\title{
Solution of the Fractional Form of Unsteady Squeezing Flow through Porous Medium
}

\author{
A. A. Hemeda, E. E. Eladdad, and I. A. Lairje \\ Department of Mathematics, Faculty of Science, Tanta University, Tanta 31527, Egypt \\ Correspondence should be addressed to A. A. Hemeda; aahemeda@yahoo.com
}

Received 21 March 2017; Revised 17 May 2017; Accepted 15 June 2017; Published 1 August 2017

Academic Editor: Mauro Gaggero

Copyright ( 2017 A. A. Hemeda et al. This is an open access article distributed under the Creative Commons Attribution License, which permits unrestricted use, distribution, and reproduction in any medium, provided the original work is properly cited.

\begin{abstract}
We propose two friendly analytical techniques called Adomian decomposition and Picard methods to analyze an unsteady axisymmetric flow of nonconducting, Newtonian fluid. This fluid is assumed to be squeezed between two circular plates passing through porous medium channel with slip and no-slip boundary conditions. A single fractional order nonlinear ordinary differential equation is obtained by means of similarity transformation with the help of the fractional calculus definitions. The resulting fractional boundary value problems are solved by the proposed methods. Convergence of the two methods' solutions is confirmed by obtaining various approximate solutions and various absolute residuals for different values of the fractional order. Comparison of the results of the two methods for different values of the fractional order confirms that the proposed methods are in a well agreement and therefore they can be used in a simple manner for solving this kind of problems. Finally, graphical study for the longitudinal and normal velocity profiles is obtained for various values of some dimensionless parameters and fractional orders.
\end{abstract}

\section{Introduction}

The importance of the fluid flow through a porous medium goes back a long time in various applications in life such as agriculture, industry, and oil and gas production, where the focus was on estimating and optimizing production. Similarly, another important application is the simulation of ground water pollution, mostly occurring due to leakage of chemicals from tanks and oil pipelines. The objective is to consider ground water as one medium and polluted water as another, so that the spreading in the latter medium and its consequences can be studied.

In recent times, after the introduction of the modified Darcy Law [1], analysis through a porous medium has advantage in an important topic for the research community such as reservoir, petroleum, chemical, civil, environmental, and agricultural and biomedical engineering. Some practical applications in these fields include chemical reactors, filtration, geothermal reservoirs, ground water hydrology, and drainage and recovery of crude oil from pores of reservoir rocks [2-7].
The importance of squeezing flow is due to its wide applications in many fields such as chemical, biomechanics, food industries, and mechanical and industrial engineering. Practical applications of squeezing flows in these fields are modeling of lubrication systems, polymer processing, compression and injection molding, and so on. These flows are induced by applying normal stresses or vertical velocities by means of a moving boundary, which can be frequently observed in various hydrodynamical tools and machines. Stefan [8] is one of the first researchers who operated on the development of squeezing flows and an ad hoc asymptotic solution to Newtonian fluids. In [9] Thorpe has established an explicit solution to the squeeze flow considering inertial terms. However, in [10], P. S. Gupta and A. S. Gupta proved that the solution given in [9] fails in satisfying boundary conditions. Verma in [11] and Singh et al. in [12] have presented numerical solutions for squeezing flow between parallel plates. Leider and Byron performed in [13] theoretical analysis of power-law fluid between parallel disks. The optimal homotopy asymptotic method (OHAM) has been used by Qayyum et al. [14] to analyze the unsteady axisymmetric 
flow of nonconducting Newtonian fluid squeezed between two circular plates with slip and no-slip boundaries. Also, the homotopy perturbation method (HPM) has been developed by Qayyum et al. [15], to model and analyze the unsteady axisymmetric flow of nonconducting, Newtonian fluid squeezed between two circular plates passing through a porous medium channel with slip boundary condition. The new iterative and Picard methods had been used by Hemeda and Eladdad in [16] for solving the fractional form of unsteady axisymmetric flow of a nonconducting, Newtonian fluid squeezed between two circular plates with slip and no-slip boundaries. For more studies on squeezing flow through porous medium and also for more theoretical and experimental studies on squeezing flow, you can see [17-25].

Fractional calculus becomes one of the important branches of applied mathematics that deals with derivatives and integrals of arbitrary orders. It is the generalized differential and integral calculus of an arbitrary order. Recently, it has the importance of many researchers because of its wide appearance in many applications in fluid mechanics, viscoelasticity, biology, physics, and engineering. Moreover, the ordinary differential operator is a local operator, but the fractional order differential operator is nonlocal. Consequently, attention has been given to the solutions of fractional differential equations for the most important fields of physics, fluid mechanics, and so on. The exact analytical solutions to most nonlinear fractional order problems cannot be found. Therefore, approximation and numerical techniques can be used. Picard method [26-29] and the Adomian decomposition method [30-34] are two powerful approaches of these techniques which can be used in simple manner and short time to obtain analytical approximations to nonlinear problems and they are particularly valuable as tools for researchers, because they provide immediate and visible symbolic terms of analytic solutions, as well as numerical approximate solutions to nonlinear differential equations without linearization or discretization.

In the last years, the applications of the proposed methods are extended to the fractional differential equations. Our objective of this article is to prepare and utilize the proposed methods to obtain an analytical solution to the fractional form of an unsteady axisymmetric flow of nonconducting, Newtonian fluid squeezed between two circular plates passing through porous medium with slip and no-slip boundary conditions. Validity of the two methods is confirmed by comparing the obtained results. In addition, the effects of various fractional order, constant containing permeability, Reynolds number, and slip parameter on the solution are studied tabularly and graphically.

\section{Formulation of the Problem}

In this section, the unsteady axisymmetric squeezing flow of incompressible first-grade fluid with density $\rho$, viscosity $\mu$, and kinematic viscosity $\nu$, squeezed between two circular plates having speed $E_{\omega}(t)$ and passing through porous medium channel, is considered with a fractional order form. At any time $t$, it is assumed that the distance between the two circular plates is $2 h(t)$. Also, it is assumed that $r$-axis is the central axis of the channel while $z$-axis is taken as normal to it. Plates move symmetrically with respect to the central axis $z=0$ while the flow is axisymmetric about $r=0$. The longitudinal and normal velocity components in radial and axial directions are $w_{r}(r, z, t)$ and $w_{z}(r, z, t)$, respectively. For more physical explanation, see [14-16].

The basic system of equations describing the motion of the fluid is

$$
\begin{aligned}
\frac{\partial w_{r}}{\partial r}+\frac{w_{r}}{r}+\frac{\partial w_{z}}{\partial z} & =0 \\
\frac{\partial p}{\partial r}+\rho\left(\frac{\partial w_{r}}{\partial t}-w_{z} \Omega\right) & =-\mu\left(\frac{\partial \Omega}{\partial z}+\frac{w_{r}}{k}\right) \\
\frac{\partial p}{\partial z}+\rho\left(\frac{\partial w_{r}}{\partial t}+w_{r} \Omega\right) & =\mu\left(\frac{1}{r} \frac{\partial}{\partial r}(r \Omega)-\frac{w_{z}}{k}\right)
\end{aligned}
$$

where $\Omega=\Omega(r, z, t)$ is the function of velocity, $p=$ $p(r, z, t)$ is the function of generalized pressure, and $k$ is the permeability constant.

The boundary values on $w_{r}(r, z, t)$ and $w_{z}(r, z, t)$ are

$$
\begin{aligned}
w_{r}(r, z, t) & =\beta \frac{\partial}{\partial z} w_{r}(r, z, t), & \\
w_{z}(r, z, t) & =E_{\omega}(t), & \\
\frac{\partial}{\partial z} w_{r}(r, z, t) & =0, & \text { at } z=h, \\
w_{z}(r, z, t) & =0, &
\end{aligned}
$$

$$
\text { at } z=0 \text {, }
$$

where $E_{\omega}(t)=d h / d t$ is the plates velocity. The boundary values in (2) are due to slipping at the upper plate when $z=h$ and symmetry at $z=0$. If we introduce the dimensionless parameter

$$
\eta=\frac{z}{h(t)}
$$

(1) transforms to

$$
\begin{aligned}
\frac{\partial w_{r}}{\partial r}+\frac{w_{r}}{r}+\frac{1}{h} \frac{\partial w_{z}}{\partial \eta} & =0 \\
\frac{\partial p}{\partial r}+\rho\left(\frac{\partial w_{r}}{\partial t}-w_{z} \Omega\right) & =-\mu\left(\frac{1}{h} \frac{\partial \Omega}{\partial \eta}+\frac{w_{r}}{k}\right), \\
\frac{1}{h} \frac{\partial p}{\partial \eta}+\rho\left(\frac{\partial w_{z}}{\partial t}+w_{r} \Omega\right) & =\mu\left(\frac{1}{r} \frac{\partial}{\partial r}(r \Omega)-\frac{w_{z}}{k}\right) .
\end{aligned}
$$

The boundary conditions on $w_{r}$ and $w_{z}$ are

$$
\begin{aligned}
& w_{r}=\beta \frac{1}{h} \frac{\partial w_{r}}{\partial \eta}, \\
& w_{z}=E_{\omega}(t),
\end{aligned}
$$

at $\eta=1$, 


$$
\begin{aligned}
\frac{\partial w_{r}}{\partial \eta} & =0, \\
w_{z} & =0,
\end{aligned}
$$

$$
\text { at } \eta=0 \text {. }
$$

The elimination of $p$ between (5) and (6) gives

$$
\begin{gathered}
\rho\left[\frac{\partial \Omega}{\partial t}+w_{r} \frac{\partial \Omega}{\partial r}+\frac{w_{z}}{h} \frac{\partial \Omega}{\partial \eta}-\frac{w_{r}}{r} \Omega\right] \\
=\mu\left[\nabla^{2} \Omega-\left(\frac{1}{r^{2}}+\frac{1}{k}\right) \Omega\right],
\end{gathered}
$$

where $\nabla^{2}$ is the Laplacian operator.

Defining the components of velocity as follows [10]:

$$
\begin{aligned}
& w_{r}=-\frac{r}{2 h(t)} E_{\omega}(t) u^{\prime}(\eta), \\
& w_{z}=E_{\omega}(t) u(\eta),
\end{aligned}
$$

we see that (4) is identically satisfied and (8) becomes

$$
\begin{aligned}
& \frac{d^{4} u}{d \eta^{4}}+R\left[(\eta-u) \frac{d^{3} u}{d \eta^{3}}+2 \frac{d^{2} u}{d \eta^{2}}\right]-Q \frac{d^{2} u}{d \eta^{2}}-M \frac{d^{2} u}{d \eta^{2}} \\
& \quad=0,
\end{aligned}
$$

where

$$
\begin{aligned}
R & =\frac{h E_{\omega}(t)}{v}, \\
Q & =\frac{h^{2}}{v E_{\omega}} \frac{d E_{\omega}(t)}{d t}, \\
M & =\frac{h^{2}}{k} .
\end{aligned}
$$

In the above equations $R$ and $Q$ are functions of $t$ but, for similarity solution, we consider them as constants. Since $E_{\omega}=$ $d h / d t$, integrating first equation of (11), we get

$$
h(t)=(c t+d)^{1 / 2},
$$

where $c$ and $d$ are constants. The plates move away from each other symmetrically with respect to $\eta$ when $c>0$ and $d>$ 0 . The squeezing flow exists when the plates approach each other when $c<0$, $d>0$ and $h(t)>0$. From (11) and (12) it follows that $Q=-R$. Then (10) becomes

$$
\frac{d^{4} u}{d \eta^{4}}+R\left[(\eta-u) \frac{d^{3} u}{d \eta^{3}}+3 \frac{d^{2} u}{d \eta^{2}}\right]-M \frac{d^{2} u}{d \eta^{2}}=0
$$

Using (7) and (9), the boundary conditions in cases of no-slip and slip boundaries at the upper plate can be defined in the form:

$$
\begin{gathered}
u(1)=1, \\
u^{\prime}(1)=0,
\end{gathered}
$$

$$
\begin{aligned}
u(0) & =0, \\
u^{\prime \prime}(0) & =0,
\end{aligned}
$$

(No-slip at the wall),

$$
\begin{aligned}
u(1) & =1, \\
u^{\prime}(1) & =\gamma u^{\prime \prime}(1), \\
u(0) & =0, \\
u^{\prime \prime}(0) & =0,
\end{aligned}
$$

(Slip at the wall).

\section{Fractional Calculus}

In the following, we state some definitions of the fractional calculus, which can be used in this work.

Definition 1. The Riemann-Liouville fractional integral operator of order $\alpha>0$ and of a function $g(t) \in c_{\mu}$ and $\mu \geq-1$ is defined as follows [35]:

$$
\begin{aligned}
& I_{t}^{\alpha} g(t)=\frac{1}{\Gamma(\alpha)} \int_{0}^{t}(t-\tau)^{\alpha-1} g(\tau) d \tau, \quad \alpha>0, t>0, \\
& I_{t}^{0} g(t)=g(t) .
\end{aligned}
$$

Also, for $I_{t}^{\alpha}$, we can define

$$
I_{t}^{\alpha} t^{v}=\frac{\Gamma(v+1) t^{v+\alpha}}{\Gamma(v+1+\alpha)} .
$$

Definition 2. The fractional order derivative $D_{t}^{\alpha}$ of $g(t)$ in the Caputo sense is defined as follows [36]:

$$
\begin{aligned}
D_{t}^{\alpha} g(t) & =I_{t}^{m-\alpha} D_{t}^{m} g(t) \\
& =\frac{1}{\Gamma(m-\alpha)} \int_{0}^{t}(t-\tau)^{m-\alpha-1} g^{(m)}(\tau) d \tau, \\
& \quad \text { for } m-1<\alpha \leq m, t>0 .
\end{aligned}
$$

For the Caputo fractional derivative operator, $D_{t}^{\alpha}$, we obtain

$$
D_{t}^{\alpha} t^{v}=\frac{\Gamma(v+1) t^{v-\alpha}}{\Gamma(v+1-\alpha)}
$$

For the Riemann-Liouvill fractional integral and Caputo fractional derivative of order $\alpha$, we have the following relations. 
Lemma 3. If $m-1<\alpha \leq m, m \in N$, and $g \in C_{\mu}^{m}, \mu \geq-1$, then

$$
\begin{aligned}
& D_{t}^{\alpha} I_{t}^{\alpha} g(t)=g(t), \\
& I_{t}^{\alpha} D_{t}^{\alpha} g(t) \\
& = \begin{cases}g(t)-\sum_{k=0}^{m-1} g^{(k)}\left(0_{+}\right) \frac{t^{k}}{k !}, & t>0, m-1<\operatorname{deg} \cdot g(t), \\
0, & m-1 \geq \operatorname{deg} \cdot g(t) .\end{cases}
\end{aligned}
$$

Remark 4. According to the above definitions of the fractional calculus, (13) can be rewritten in the fractional order form:

$$
\begin{aligned}
\frac{d^{\alpha} u}{d \eta^{\alpha}}+R\left[(\eta-u) \frac{d^{3} u}{d \eta^{3}}+3 \frac{d^{2} u}{d \eta^{2}}\right]-M \frac{d^{2} u}{d \eta^{2}} & =0, \\
& 3<\alpha \leq 4 .
\end{aligned}
$$

\section{Analysis of the Proposed Methods}

In this section, we present the analysis of the proposed methods with their suitable algorithms for the fractional order problems.

4.1. Picard Method (PM). To present this method, let us consider the general fractional order problem with an arbitrary order $\alpha>0$ [26-29]:

$$
\begin{aligned}
& D_{t}^{\alpha} u(t)=F\left(t, u^{(k)}(t)\right), \\
& \qquad m-1<\alpha \leq m, m \in N, \\
& \frac{d^{k}}{d t^{k}} u(0)=h_{k}, \quad k=0,1,2, \ldots, m-1,
\end{aligned}
$$

where $D_{t}^{\alpha}$ is the time fractional differential operator of order $\alpha>0$. According to the fractional integral operators, the fractional order problem (21a) and (21b) takes the equivalent fractional integral form:

$$
u(t)=\sum_{k=0}^{m-1} h_{k} \cdot \frac{t^{k}}{k !}+I_{t}^{\alpha}\left[F\left(t, u_{t}^{(k)}(t)\right)\right]=f+N(u),
$$

where $f=\sum_{k=0}^{m-1} h_{k} \cdot t^{k} / k !, N(u)=I_{t}^{\alpha}\left[F\left(t, u_{t}^{(k)}(t)\right)\right]$, and $I_{t}^{\alpha}$ is the inverse of $D_{t}^{\alpha}$. The required solution $u(t)$ for (22) which is also the solution for (21a) and (21b) can be obtained as the limit of a sequence of functions $u_{r+1}(t)$ generated by the recurrence relation:

$$
\begin{aligned}
u_{0} & =f, \\
u_{r+1} & =u_{0}+N\left(u_{r}\right), \quad r=0,1,2, \ldots,
\end{aligned}
$$

where $u(t)=\lim _{r \rightarrow \infty} u_{r}(t)$.

4.2. Adomian Decomposition Method (ADM). To illustrate the idea of this method, let us consider the fractional order problem [30-34]:

$$
\begin{aligned}
D_{t}^{\alpha} u(t)=L u(t)+N u(t)+ & g(t), \\
& m-1<\alpha \leq m, m \in N,
\end{aligned}
$$

subject to the initial values:

$$
\frac{d^{k}}{d t^{k}} u(0)=h_{k}, \quad k=0,1,2, \ldots, m-1,
$$

where $L, N$ are linear and nonlinear operators and $g(t)$ is a nonhomogeneous term. The method is based on applying the fractional integral operator $I_{t}^{\alpha}$, the inverse of the fractional differential operator $D_{t}^{\alpha}$, to both sides of (24a) and (24b) to obtain

$$
u(t)=\sum_{k=0}^{m-1} h_{k} \cdot \frac{t^{k}}{k !}+I_{t}^{\alpha}[L u(t)+N u(t)+g(t)] .
$$

The ADM suggests that the solution $u(t)$ be decomposed into the infinite series of components:

$$
u(t)=\sum_{n=0}^{\infty} u_{n}(t)
$$

and the nonlinear term $N u$ in $(24 a)$ is decomposed as

$$
N u=\sum_{n=0}^{\infty} A_{n}
$$

where $A_{n}$ are called Adomian polynomials. Substituting the decomposition series (26) and (27) into both sides of (25) gives

$$
\begin{aligned}
\sum_{n=0}^{\infty} u_{n}(t)= & \sum_{k=0}^{m-1} h_{k} \cdot \frac{t^{k}}{k !} \\
& +I_{t}^{\alpha}\left[L \sum_{n=0}^{\infty} u_{n}(t)+\sum_{n=0}^{\infty} A_{n}+g(t)\right] .
\end{aligned}
$$

Following the decomposition method, we introduce the recurrence relation as

$$
\begin{aligned}
u_{0}(t) & =\sum_{k=0}^{m-1} h_{k} \cdot \frac{t^{k}}{k !}+I_{t}^{\alpha}[g(t)], \\
u_{j+1}(t) & =I_{t}^{\alpha}\left[L u_{j}(t)+A_{j}\right], \quad j \geq 0 .
\end{aligned}
$$

The Adomian polynomial $A_{n}$ can be calculated for all forms of nonlinearity according to specific algorithms constructed by Adomian. The general form of the Adomian polynomials formula for $A_{n}$ is

$$
A_{n}=\frac{1}{n !}\left[\frac{d^{n}}{d \lambda^{n}} N\left(\sum_{k=0}^{n} \lambda^{k} u_{k}\right)\right]_{\lambda=0} .
$$

This formula is easy to compute. Finally, we approximate the solution $u(t)$ by the truncated series:

$$
\begin{aligned}
\phi_{N}(t) & =\sum_{j=0}^{N-1} u_{j}(t), \\
\lim _{N \rightarrow \infty} \phi_{N}(t) & =u(t) .
\end{aligned}
$$




\section{Applications}

In this section, we illustrate the application of the two proposed methods to solve the nonlinear fractional order ordinary differential equation (20) subject to the boundary conditions (14a) and (14b).

5.1. PM. Using (20) and (14a) and (14b), the initial value fractional order problem

$$
\begin{aligned}
\frac{d^{\alpha}}{d t^{\alpha}} u(\eta)+R\left[(\eta-u) \frac{d^{3} u}{d \eta^{3}}+3 \frac{d^{2} u}{d \eta^{2}}\right]-M \frac{d^{2} u}{d \eta^{2}} & =0, \\
3<\alpha \leq 4 & \\
u(0) & =0, \\
u^{\prime}(0) & =a, \\
u^{\prime \prime}(0) & =0, \\
u^{\prime \prime \prime}(0) & =b
\end{aligned}
$$

according to (22) and (23), becomes

$$
\begin{aligned}
& u_{n+1}(\eta) \\
& =u_{0}(\eta) \\
& \quad-I_{\eta}^{\alpha}\left[R\left(\left(\eta-u_{n}\right) \frac{d^{3} u_{n}}{d \eta^{3}}+3 \frac{d^{2} u_{n}}{d \eta^{2}}\right)-M \frac{d^{2} u_{n}}{d \eta^{2}}\right], \\
& u_{0}(\eta)=a \eta+\frac{b \eta^{3}}{6}, \quad n \geq 0 .
\end{aligned}
$$

Therefore, by Wolframe Mathematica 10 Package, we can obtain the following first few components of the solution:

$$
\begin{aligned}
& u_{0}(\eta)=a \eta+\frac{b \eta^{3}}{6}, \\
& u_{1}(\eta)=a \eta+\frac{b \eta^{3}}{6}-\frac{4 b R \eta^{1+\alpha}}{\Gamma(2+\alpha)}+\frac{a b R \eta^{1+\alpha}}{\Gamma(2+\alpha)} \\
& +\frac{b^{2} R \eta^{3+\alpha}}{\Gamma(4+\alpha)}+\frac{b M \eta^{1+\alpha}}{\Gamma(2+\alpha)}, \\
& u_{2}(\eta)=a \eta+\frac{b \eta^{3}}{6}+\frac{b M \eta^{1+\alpha}}{\Gamma(2+\alpha)}-\frac{4 b R \eta^{1+\alpha}}{\Gamma(2+\alpha)} \\
& +\frac{a b R \eta^{1+\alpha}}{\Gamma(2+\alpha)}+\frac{b^{2} R \eta^{3+\alpha}}{\Gamma(4+\alpha)}-\frac{b M R(-1+\alpha) \eta^{-1+2 \alpha}}{\Gamma(2 \alpha)} \\
& +\frac{a b M R(-1+\alpha) \eta^{-1+2 \alpha}}{\Gamma(2 \alpha)}+\frac{4 b R^{2}(-1+\alpha) \eta^{-1+2 \alpha}}{\Gamma(2 \alpha)} \\
& +\frac{5 a b R^{2}(-1+\alpha) \eta^{-1+2 \alpha}}{\Gamma(2 \alpha)}+\cdots,
\end{aligned}
$$

and so on. In the same manner, the rest of the components can be obtained. The 4 th-order term solution for (32), in series form, is given by

$$
\begin{aligned}
u_{3}(\eta)= & a \eta+\frac{b \eta^{3}}{6}+\frac{b M \eta^{1+\alpha}}{\Gamma(2+\alpha)}-\frac{4 b R \eta^{1+\alpha}}{\Gamma(2+\alpha)} \\
& +\frac{a b R \eta^{1+\alpha}}{\Gamma(2+\alpha)}+\frac{b^{2} R \eta^{3+\alpha}}{\Gamma(4+\alpha)} \\
& -\frac{b M R(-1+\alpha) \eta^{-1+2 \alpha}}{\Gamma(2 \alpha)} \\
& +\frac{a b M R(-1+\alpha) \eta^{-1+2 \alpha}}{\Gamma(2 \alpha)} \\
& +\frac{4 b R^{2}(-1+\alpha) \eta^{-1+2 \alpha}}{\Gamma(2 \alpha)} \\
& -\frac{5 a b R^{2}(-1+\alpha) \eta^{-1+2 \alpha}}{\Gamma(2 \alpha)}+\cdots
\end{aligned}
$$

In the special case, $\alpha=4$, (35) gives

$$
\begin{aligned}
u_{3}(\eta)= & a \eta+\frac{b \eta^{3}}{6}+\frac{b M \eta^{5}}{120}-\frac{b R \eta^{5}}{30}+\frac{a b R \eta^{5}}{120} \\
& +\frac{b M^{2} \eta^{7}}{5040}+\frac{b^{2} R \eta^{7}}{5040}-\frac{b M R \eta^{7}}{504}+\frac{a b M R \eta^{7}}{1260} \\
& +\frac{b R^{2} \eta^{7}}{210}-\frac{a b R^{2} \eta^{7}}{280}+\frac{a^{2} b R^{2} \eta^{7}}{1680}-\frac{b M^{3} \eta^{9}}{362880} \\
& +\cdots
\end{aligned}
$$

Using the boundary conditions in (14a) and (14b) with the initial conditions in (32), the unknowns $a$ and $b$ for fixed values of $R$ and $M$ in (36) can be easily determined. In case of no-slip boundary, then $a=1.5$ and $b=-3.0$. For $R=M=$ 0.5 , the solution is

$$
\begin{aligned}
u(\eta)= & 1.5 \eta-0.5 \eta^{3}+0.01875 \eta^{5}+0.00078125 \eta^{7} \\
& -9.95939 \times 10^{-5} \eta^{9}-4.67144 \times 10^{-6} \eta^{11} \\
& +8.14242 \times 10^{-7} \eta^{13}+2.31908 \times 10^{-8} \eta^{15} \\
& -2.27551 \times 10^{-9} \eta^{17}+7.48163 \times 10^{-12} \eta^{19} \\
& +3.60882 \times 10^{-12} \eta^{21}-1.75116 \times 10^{-15} \eta^{23} \\
& -4.14156 \times 10^{-15} \eta^{25}-1.83285 \times 10^{-17} \eta^{27} \\
& +1.31237 \times 10^{-18} \eta^{29}+1.18008 \times 10^{-20} \eta^{31}
\end{aligned}
$$

and in case of slip boundary, then $a=0.75$ and $b=1.5$. For $R=M=0.5$, the solution is

$$
\begin{aligned}
u(\eta)= & 0.75 \eta+0.25 \eta^{3}-0.0140625 \eta^{5} \\
& +0.000683594 \eta^{7}-5.37933 \times 10^{-5} \eta^{9} \\
& +3.8193 \times 10^{-6} \eta^{11}-2.57439 \times 10^{-7} \eta^{13}
\end{aligned}
$$




$$
\begin{aligned}
& +1.27696 \times 10^{-8} \eta^{15}-4.75209 \times 10^{-10} \eta^{17} \\
& +1.56632 \times 10^{-11} \eta^{19}-4.75822 \times 10^{-13} \eta^{21} \\
& +1.16642 \times 10^{-14} \eta^{23}-2.03097 \times 10^{-16} \eta^{25} \\
& +2.3071 \times 10^{-18} \eta^{27}-1.53793 \times 10^{-20} \eta^{29} \\
& +4.6097 \times 10^{-23} \eta^{31} .
\end{aligned}
$$

$$
\begin{aligned}
& -\frac{5 a b R^{2}(\alpha-1) \eta^{-1+2 \alpha}}{\Gamma(2 \alpha)}+\frac{a^{2} b R^{2}(-1+\alpha) \eta^{-1+2 \alpha}}{\Gamma(2 \alpha)} \\
& +\frac{b^{2} M R \Gamma(2+\alpha) \eta^{1+2 \alpha}}{6 \Gamma(-1+\alpha) \Gamma(2+2 \alpha)}-\cdots,
\end{aligned}
$$

5.2. ADM. According to the recurrence relation (29), the initial value fractional order problem (32) gives

$$
\begin{aligned}
& u_{0}(\eta)=a \eta+\frac{b \eta^{3}}{6} \\
& u_{j+1}(\eta) \\
& =-I_{t}^{\alpha}\left[R\left(\eta \frac{d^{3} u_{j}}{d \eta^{3}}+3 \frac{d^{2} u_{j}}{d \eta^{2}}-A_{j}\right)-M \frac{d^{2} u_{j}}{d \eta^{2}}\right],
\end{aligned}
$$

$$
j \geq 0 \text {, }
$$

where

$$
\begin{aligned}
& A_{0}=u_{0} \frac{d^{3} u_{0}}{d \eta^{3}} \\
& A_{1}=u_{0} \frac{d^{3} u_{1}}{d \eta^{3}}+u_{1} \frac{d^{3} u_{0}}{d \eta^{3}} \\
& A_{2}=u_{0} \frac{d^{3} u_{2}}{d \eta^{3}}+u_{1} \frac{d^{3} u_{1}}{d \eta^{3}}+u_{2} \frac{d^{3} u_{0}}{d \eta^{3}}, \\
& A_{3}=u_{0} \frac{d^{3} u_{3}}{d \eta^{3}}+u_{1} \frac{d^{3} u_{2}}{d \eta^{3}}+u_{2} \frac{d^{3} u_{1}}{d \eta^{3}}+u_{3} \frac{d^{3} u_{0}}{d \eta^{3}}, \ldots,
\end{aligned}
$$

and, therefore, the first few components of the solution are as follows:

$$
\begin{aligned}
& u_{0}(\eta)=a \eta+\frac{b \eta^{3}}{6}, \\
& u_{1}(\eta)=-\frac{4 b R \eta^{1+\alpha}}{\Gamma(2+\alpha)}+\frac{a b R \eta^{1+\alpha}}{\Gamma(2+\alpha)}+\frac{b^{2} R \eta^{3+\alpha}}{\Gamma(4+\alpha)} \\
& +\frac{b M \eta^{1+\alpha}}{\Gamma(2+\alpha)}, \\
& u_{2}(\eta)=-\frac{b M R(-1+\alpha) \eta^{-1+2 \alpha}}{\Gamma(2 \alpha)} \\
& +\frac{a b M R(-1+\alpha) \eta^{-1+2 \alpha}}{\Gamma(2 \alpha)}+\frac{4 b R^{2}(\alpha-1) \eta^{-1+2 \alpha}}{\Gamma(2 \alpha)}
\end{aligned}
$$

and so on. In the same manner the rest of the components can be obtained. The 4-term solution is

$$
\begin{aligned}
u(\eta)= & \sum_{i=0}^{3} u_{i}(\eta) \\
= & a \eta+\frac{b \eta^{3}}{6}-\frac{4 b R \eta^{1+\alpha}}{\Gamma(2+\alpha)}+\frac{a b R \eta^{1+\alpha}}{\Gamma(2+\alpha)}+\frac{b^{2} R \eta^{3+\alpha}}{\Gamma(4+\alpha)} \\
& +\frac{b M \eta^{1+\alpha}}{\Gamma(2+\alpha)}-\frac{b M R(-1+\alpha) \eta^{-1+2 \alpha}}{\Gamma(2 \alpha)} \\
& +\frac{a b M R(-1+\alpha) \eta^{-1+2 \alpha}}{\Gamma(2 \alpha)} \\
& +\frac{4 b R^{2}(-1+\alpha) \eta^{-1+2 \alpha}}{\Gamma(2 \alpha)} \\
& -\frac{5 a b R^{2}(-1+\alpha) \eta^{-1+2 \alpha}}{\Gamma(2 \alpha)} \\
& +\frac{a^{2} b R^{2}(-1+\alpha) \eta^{-1+2 \alpha}}{\Gamma(2 \alpha)} \\
& +\frac{b^{2} M R \Gamma(2+\alpha) \eta^{1+2 \alpha}}{6 \Gamma(-1+\alpha) \Gamma(2+2 \alpha)}+\cdots
\end{aligned}
$$

In the special case, $\alpha=4$, (42) gives

$$
\begin{aligned}
u(\eta)= & a \eta+\frac{b \eta^{3}}{6}+\frac{b M \eta^{5}}{120}-\frac{b R \eta^{5}}{30}+\frac{a b R \eta^{5}}{120} \\
& +\frac{b M^{2} \eta^{7}}{5040}+\frac{b^{2} R \eta^{7}}{5040}-\frac{b M R \eta^{7}}{504}+\frac{a b M R \eta^{7}}{1260} \\
& +\frac{b R^{2} \eta^{7}}{210}-\frac{a b R^{2} \eta^{7}}{280}+\frac{a^{2} b R^{2} \eta^{7}}{1680}-\frac{b M^{3} \eta^{9}}{362880} \\
& +\cdots
\end{aligned}
$$

Similarly, using the boundary conditions in (14a) and (14b) with the initial conditions in (32), the solution in case of noslip boundary, for $R=M=0.5$, is

$$
\begin{aligned}
u(\eta)= & 1.5 \eta-0.5 \eta^{3}+0.01875 \eta^{5}+0.00078125 \eta^{7} \\
& -9.95939 \times 10^{-5} \eta^{9}-4.67144 \times 10^{-6} \eta^{11} \\
& +8.20035 \times 10^{-7} \eta^{13}+4.88258 \times 10^{-8} \eta^{15}
\end{aligned}
$$


TABLE 1: Solutions for different values of $\eta$ and $\alpha$ at $R=0.5, M=0.3$, in case of no-slip boundary, by the two methods.

\begin{tabular}{|c|c|c|c|c|c|c|}
\hline \multirow{2}{*}{$\eta$} & \multicolumn{3}{|c|}{$\mathrm{PM}$} & \multicolumn{3}{|c|}{$\mathrm{ADM}$} \\
\hline & $\alpha=3.9$ & $\alpha=3.95$ & $\alpha=4.0$ & $\alpha=4.0$ & $\alpha=3.95$ & $\alpha=3.9$ \\
\hline 0.0 & 0.0 & 0.0 & 0.0 & 0.0 & 0.0 & 0.0 \\
\hline 0.1 & 0.149500 & 0.149500 & 0.149500 & 0.149500 & 0.149500 & 0.149500 \\
\hline 0.2 & 0.296011 & 0.296009 & 0.296008 & 0.296008 & 0.296009 & 0.296011 \\
\hline 0.3 & 0.436577 & 0.436567 & 0.436558 & 0.436558 & 0.436567 & 0.436577 \\
\hline 0.4 & 0.568317 & 0.568278 & 0.568244 & 0.568244 & 0.568278 & 0.568316 \\
\hline 0.5 & 0.688448 & 0.688342 & 0.688247 & 0.688247 & 0.688340 & 0.688445 \\
\hline 0.6 & 0.794321 & 0.794080 & 0.793863 & 0.793863 & 0.794074 & 0.794313 \\
\hline 0.7 & 0.883451 & 0.882973 & 0.882539 & 0.882539 & 0.882955 & 0.883428 \\
\hline 0.8 & 0.953549 & 0.952688 & 0.951899 & 0.951899 & 0.952642 & 0.953492 \\
\hline 0.9 & 1.002550 & 1.001110 & 0.999779 & 0.999779 & 1.001000 & 1.002420 \\
\hline 1.0 & 1.028640 & 1.026370 & 1.024260 & 1.024260 & 1.026150 & 1.028380 \\
\hline
\end{tabular}

TABLE 2: Solutions for different values of $\eta$ and $\alpha$ at $R=0.3, M=0.5$, in case of no-slip boundary, by the two methods.

\begin{tabular}{|c|c|c|c|c|c|c|}
\hline \multirow{2}{*}{$\eta$} & \multicolumn{3}{|c|}{$\mathrm{PM}$} & \multicolumn{3}{|c|}{$\mathrm{ADM}$} \\
\hline & $\alpha=3.9$ & $\alpha=3.95$ & $\alpha=4.0$ & $\alpha=4.0$ & $\alpha=3.95$ & $\alpha=3.9$ \\
\hline 0.0 & 0.0 & 0.0 & 0.0 & 0.0 & 0.0 & 0.0 \\
\hline 0.1 & 0.149500 & 0.149500 & 0.149500 & 0.149500 & 0.149500 & 0.149500 \\
\hline 0.2 & 0.296003 & 0.296002 & 0.296002 & 0.296002 & 0.296002 & 0.296003 \\
\hline 0.3 & 0.436520 & 0.436518 & 0.436515 & 0.436515 & 0.436518 & 0.436520 \\
\hline 0.4 & 0.568084 & 0.568074 & 0.568065 & 0.568065 & 0.568074 & 0.568084 \\
\hline 0.5 & 0.687754 & 0.687725 & 0.687700 & 0.687700 & 0.687724 & 0.687753 \\
\hline 0.6 & 0.792625 & 0.792560 & 0.792501 & 0.792501 & 0.792557 & 0.792622 \\
\hline 0.7 & 0.879845 & 0.879714 & 0.879594 & 0.879594 & 0.879706 & 0.8879835 \\
\hline 0.8 & 0.946620 & 0.946379 & 0.946159 & 0.946159 & 0.946359 & 0.946595 \\
\hline 0.9 & 0.990229 & 0.989819 & 0.989443 & 0.989443 & 0.989773 & 0.990172 \\
\hline 1.0 & 1.008040 & 1.007380 & 1.006770 & 1.006770 & 1.007290 & 1.007920 \\
\hline
\end{tabular}

and in case of slip boundary, for $R=M=0.5$, it is

$$
\begin{aligned}
u(\eta)= & 0.75 \eta+0.25 \eta^{3}-0.0140625 \eta^{5} \\
& +0.000683594 \eta^{7}-5.37933 \times 10^{-5} \eta^{9} \\
& +3.81932 \times 10^{-6} \eta^{11}-1.96303 \times 10^{-7} \eta^{13} \\
& +3.05161 \times 10^{-9} \eta^{15}
\end{aligned}
$$

It is clear that the number of terms of the solution obtained by ADM in (42), (43), (44), and (45) is less than the number obtained by PM in (35), (36), (37), and (38).

The residual error of the problem is

$$
\begin{aligned}
\operatorname{Re}(\eta) & =\text { Residual Error } \\
& =\frac{d^{\alpha} \widehat{u}}{d \eta^{\alpha}}+R\left[(\eta-\widehat{u}) \frac{d^{3} \widehat{u}}{d \eta^{3}}+3 \frac{d^{2} \widehat{u}}{d \eta^{2}}\right]-M \frac{d^{2} \widehat{u}}{d \eta^{2}},
\end{aligned}
$$

where $\widehat{u}$ is the 4-term approximate solution in (35) or (42) for (32).

It is important to note that, when $\operatorname{Re}=0, \widehat{u}$ will be the exact solution. But, in nonlinear problems, this case does not usually occur.
It is clear from the obtained results that the above considered methods are used simply and accurately without linearization or discretization with their difficulties. Therefore, these methods are powerful methods for solving the nonlinear fractional order differential equations.

\section{Numerical Results and Discussion}

In this work, an unsteady axisymmetric flow of incompressible, nonconducting Newtonian fluid squeezed between two circular plates passing through porous medium channel with slip and no-slip boundary conditions is considered. The obtained nonlinear fractional order problems are solved analytically through PM and ADM using Wolframe Mathematica 10 Package.

In addition to the fractional order $\alpha$, there are three parameters: constant containing permeability $M$, Reynolds number $R$, and slip parameter $\gamma$ in the considered problem. Our discussions of the obtained results are based on different values of $\alpha$ and these parameters. First, in case of no-slip boundary, the 4 th-order approximate solutions obtained by the two methods for different values of $\alpha, R$, and $M$ are illustrated in Tables 1 and 2. Secondly, Tables 3 and 4 represent the 4 th-order absolute residual errors |Re| for different values 
TABLE 3: Fourth-order absolute residual errors $|\mathrm{Re}|$ for different values of $\eta, \alpha$ at $R=0.3, M=0.5$ in case of no-slip boundary, by the two methods.

\begin{tabular}{|c|c|c|c|c|c|c|}
\hline \multirow{2}{*}{$\eta$} & \multicolumn{3}{|c|}{$\mathrm{PM}$} & \multicolumn{3}{|c|}{$\mathrm{ADM}$} \\
\hline & $\alpha=3.9$ & $\alpha=3.95$ & $\alpha=4.0$ & $\alpha=4.0$ & $\alpha=3.95$ & $\alpha=3.9$ \\
\hline 0.0 & 0.0 & 0.0 & 0.0 & 0.0 & 0.0 & 0.0 \\
\hline 0.1 & $1.34647^{-9}$ & $1.17813^{-9}$ & $9.87225^{-10}$ & $1.05780^{-9}$ & $1.27939^{-9}$ & $1.49156^{-9}$ \\
\hline 0.2 & $2.09141^{-8}$ & $2.24087^{-8}$ & $2.18121^{-8}$ & $3.09468^{-8}$ & $3.46406^{-8}$ & $3.72693^{-8}$ \\
\hline 0.3 & $2.76031^{-8}$ & $2.33410^{-8}$ & $5.34534^{-8}$ & $2.12433^{-7}$ & $2.27849^{-7}$ & $2.35087^{-7}$ \\
\hline 0.4 & $9.08772^{-7}$ & $5.72543^{-7}$ & $3.30248^{-7}$ & $8.91625^{-7}$ & $9.55578^{-7}$ & $9.99600^{-7}$ \\
\hline 0.5 & $4.75214^{-6}$ & $3.54731^{-6}$ & $2.60346^{-6}$ & $3.41554^{-6}$ & $3.81990^{-6}$ & $4.25255^{-6}$ \\
\hline 0.6 & $1.40546^{-5}$ & $1.12830^{-5}$ & $8.97699^{-6}$ & $1.34531^{-5}$ & $1.57013^{-5}$ & $1.83641^{-5}$ \\
\hline 0.7 & $2.48516^{-5}$ & $2.13018^{-5}$ & $1.80758^{-5}$ & $5.09950^{-5}$ & $6.06163^{-5}$ & $7.21757^{-5}$ \\
\hline 0.8 & $6.28431^{-6}$ & $9.05690^{-6}$ & $1.05177^{-5}$ & $1.74732^{-4}$ & $2.08025^{-4}$ & $2.47783^{-4}$ \\
\hline 0.9 & $1.46521^{-4}$ & $1.12844^{-4}$ & $8.62395^{-5}$ & $5.33866^{-4}$ & $6.32037^{-4}$ & $7.48016^{-4}$ \\
\hline 1.0 & $7.06754^{-4}$ & $5.77755^{-4}$ & $4.70915^{-4}$ & $1.46811^{-3}$ & $1.72421^{-3}$ & $2.02338^{-3}$ \\
\hline
\end{tabular}

TABLE 4: Fourth-order absolute residual errors $|\operatorname{Re}|$ for different values of $\eta, \alpha$ at $R=0.3, M=0.7$ in case of no-slip boundary, by the two methods.

\begin{tabular}{lcccccc}
\hline$\eta$ & & PM & & & \multicolumn{2}{c}{ ADM } \\
& $\alpha=3.9$ & $\alpha=3.95$ & $\alpha=4.0$ & $\alpha=4.0$ & $\alpha=3.95$ & 0.0 \\
\hline 0.0 & 0.0 & 0.0 & 0.0 & 0.0 & $1.69724^{-9}$ & $2.38949^{-9}$ \\
0.1 & $3.34624^{-9}$ & $2.38537^{-9}$ & $1.69437^{-9}$ & $5.21676^{-8}$ & $6.85643^{-8}$ & $3.35214^{-9}$ \\
0.2 & $8.91047^{-8}$ & $6.80440^{-8}$ & $5.17797^{-8}$ & $3.98014^{-8}$ \\
0.3 & $5.71570^{-7}$ & $4.54449^{-7}$ & $3.60082^{-7}$ & $5.6323^{-7}$ & $4.63794^{-7}$ & $1.67684^{-6}$ \\
0.4 & $1.95326^{-6}$ & $1.60024^{-6}$ & $1.30642^{-6}$ & $1.36736^{-6}$ & $2.04942^{-6}$ \\
0.5 & $4.40399^{-6}$ & $3.70732^{-6}$ & $3.10861^{-6}$ & $3.44222^{-6}$ & $4.11856^{-6}$ & $4.91033^{-6}$ \\
0.6 & $6.66441^{-6}$ & $5.80184^{-6}$ & $5.02294^{-6}$ & $6.41819^{-6}$ & $7.49551^{-6}$ & $8.71803^{-6}$ \\
0.7 & $4.88823^{-6}$ & $4.66370^{-6}$ & $4.36538^{-6}$ & $9.21314^{-6}$ & $1.04746^{-5}$ & $1.18463^{-5}$ \\
0.8 & $6.46530^{-6}$ & $4.52650^{-6}$ & $3.20404^{-6}$ & $1.14928^{-5}$ & $1.28039^{-5}$ & $1.41866^{-5}$ \\
0.9 & $2.64276^{-5}$ & $2.20234^{-5}$ & $1.82974^{-5}$ & $2.18120^{-5}$ & $2.51127^{-5}$ & $2.89148^{-5}$ \\
1.0 & $2.13925^{-5}$ & $2.04655^{-5}$ & $1.92411^{-5}$ & $8.14057^{-5}$ & $9.68709^{-5}$ & $1.15284^{-4}$ \\
\hline
\end{tabular}

TABLE 5: Solutions for different values of $\eta$ and $\alpha$ at $R=0.5, M=0.3$, and $\gamma=1$, in case of slip boundary, by the two methods.

\begin{tabular}{|c|c|c|c|c|c|c|}
\hline \multirow{2}{*}{$\eta$} & \multicolumn{3}{|c|}{ PM } & \multicolumn{3}{|c|}{$\mathrm{ADM}$} \\
\hline & $\alpha=3.9$ & $\alpha=3.95$ & $\alpha=4.0$ & $\alpha=4.0$ & $\alpha=3.95$ & $\alpha=3.9$ \\
\hline 0.0 & 0.0 & 0.0 & 0.0 & 0.0 & 0.0 & 0.0 \\
\hline 0.1 & 0.075250 & 0.075250 & 0.075250 & 0.075250 & 0.075250 & 0.075250 \\
\hline 0.2 & 0.151993 & 0.151994 & 0.151995 & 0.151995 & 0.151994 & 0.151993 \\
\hline 0.3 & 0.231697 & 0.231704 & 0.231710 & 0.231710 & 0.231704 & 0.231697 \\
\hline 0.4 & 0.315782 & 0.315808 & 0.315832 & 0.315832 & 0.315809 & 0.315782 \\
\hline 0.5 & 0.405603 & 0.405675 & 0.405739 & 0.405739 & 0.405676 & 0.405605 \\
\hline 0.6 & 0.502430 & 0.502590 & 0.502735 & 0.502735 & 0.502594 & 0.502435 \\
\hline 0.7 & 0.607433 & 0.607747 & 0.608033 & 0.608033 & 0.607760 & 0.607449 \\
\hline 0.8 & 0.721674 & 0.722231 & 0.722741 & 0.722741 & 0.722262 & 0.721712 \\
\hline 0.9 & 0.846091 & 0.847006 & 0.847849 & 0.847850 & 0.847075 & 0.846176 \\
\hline 1.0 & 0.981495 & 0.982910 & 0.984220 & 0.984220 & 0.983053 & 0.981668 \\
\hline
\end{tabular}

of $\alpha, R$, and $M$. Thirdly, Tables $5,6,7$, and 8 are in case of slip boundary at $\gamma=1$. Fourthly, Tables 9 and 10 and 11 and 12 represent the $3 r d$-order and 2 nd-order absolute residual errors $|\mathrm{Re}|$ for different values of $\alpha, R$, and $M$ in case of no-slip and slip boundaries with $\gamma=1$ for the two methods. Fifthly, the absolute difference between the ADM and PM solutions is illustrated in Tables 13 and 14 for various values of $\alpha, M$, and $R$ at $\gamma=1$ in case of no-slip and slip boundaries conditions. Finally, the analysis of the absolute residual errors is shown in Tables 15 and 16 for various values of $\alpha$,while keeping the 
TABLE 6: Solutions for different values of $\eta$ and $\alpha$ at $R=0.3, M=0.5$, and $\gamma=1$, in case of slip boundary, by the two methods.

\begin{tabular}{|c|c|c|c|c|c|c|}
\hline \multirow{2}{*}{$\eta$} & \multicolumn{3}{|c|}{$\mathrm{PM}$} & \multicolumn{3}{|c|}{$\mathrm{ADM}$} \\
\hline & $\alpha=3.9$ & $\alpha=3.95$ & $\alpha=4.0$ & $\alpha=4.0$ & $\alpha=3.95$ & $\alpha=3.9$ \\
\hline 0.0 & 0.0 & 0.0 & 0.0 & 0.0 & 0.0 & 0.0 \\
\hline 0.1 & 0.075250 & 0.075250 & 0.075250 & 0.075250 & 0.075250 & 0.075250 \\
\hline 0.2 & 0.151997 & 0.151998 & 0.151998 & 0.151998 & 0.151998 & 0.151997 \\
\hline 0.3 & 0.231731 & 0.231733 & 0.231736 & 0.231736 & 0.231733 & 0.231731 \\
\hline 0.4 & 0.315922 & 0.315931 & 0.315940 & 0.315940 & 0.315931 & 0.315922 \\
\hline 0.5 & 0.406017 & 0.406043 & 0.406066 & 0.406066 & 0.406044 & 0.406018 \\
\hline 0.6 & 0.503433 & 0.503492 & 0.503544 & 0.503544 & 0.503494 & 0.503436 \\
\hline 0.7 & 0.609549 & 0.609665 & 0.609770 & 0.609770 & 0.609672 & 0.609559 \\
\hline 0.8 & 0.725704 & 0.725910 & 0.726099 & 0.726099 & 0.725929 & 0.725727 \\
\hline 0.9 & 0.853188 & 0.853530 & 0.853846 & 0.853846 & 0.853572 & 0.853239 \\
\hline 1.0 & 0.993243 & 0.993778 & 0.994273 & 0.994273 & 0.993863 & 0.993346 \\
\hline
\end{tabular}

TABLE 7: Fourth-order absolute residual errors $|\mathrm{Re}|$ for different values of $\eta$ and $\alpha$, at $R=0.3, M=0.5$, and $\gamma=1$, in case of slip boundary, by the two methods.

\begin{tabular}{|c|c|c|c|c|c|c|}
\hline \multirow{2}{*}{$\eta$} & \multicolumn{3}{|c|}{ PM } & \multicolumn{3}{|c|}{$\mathrm{ADM}$} \\
\hline & $\alpha=3.9$ & $\alpha=3.95$ & $\alpha=4.0$ & $\alpha=4.0$ & $\alpha=3.95$ & $\alpha=3.9$ \\
\hline 0.0 & 0.0 & 0.0 & 0.0 & 0.0 & 0.0 & 0.0 \\
\hline 0.1 & $6.14308^{-8}$ & $4.19152^{-8}$ & $2.85428^{-8}$ & $2.84794^{-8}$ & $4.18242^{-8}$ & $6.13005^{-8}$ \\
\hline 0.2 & $1.67295^{-6}$ & $1.22330^{-6}$ & $8.92738^{-7}$ & $8.84643^{-7}$ & $1.21247^{-6}$ & $1.65847^{-6}$ \\
\hline 0.3 & $1.12755^{-5}$ & $8.58463^{-6}$ & $6.52302^{-6}$ & $6.38533^{-6}$ & $8.40763^{-6}$ & $1.10483^{-5}$ \\
\hline 0.4 & $4.24853^{-5}$ & $3.32810^{-5}$ & $2.60194^{-5}$ & $2.49943^{-5}$ & $3.20080^{-5}$ & $4.08886^{-5}$ \\
\hline 0.5 & $1.15444^{-4}$ & $9.24336^{-5}$ & $7.38637^{-5}$ & $6.90161^{-5}$ & $8.65133^{-5}$ & $1.08224^{-4}$ \\
\hline 0.6 & $2.53158^{-4}$ & $2.06288^{-4}$ & $1.67767^{-4}$ & $1.50576^{-4}$ & $1.85676^{-4}$ & $2.28478^{-4}$ \\
\hline 0.7 & $4.75190^{-4}$ & $3.92835^{-4}$ & $3.24121^{-4}$ & $2.74183^{-4}$ & $3.33889^{-4}$ & $4.05711^{-4}$ \\
\hline 0.8 & $7.89690^{-4}$ & $6.60620^{-4}$ & $5.51583^{-4}$ & $4.26322^{-4}$ & $5.14781^{-4}$ & $6.20133^{-4}$ \\
\hline 0.9 & $1.18531^{-3}$ & $1.00111^{-3}$ & $8.43922^{-4}$ & $5.63283^{-4}$ & $6.78348^{-4}$ & $8.14641^{-4}$ \\
\hline 1.0 & $1.62509^{-3}$ & $1.38247^{-3}$ & $1.17383^{-3}$ & $5.99153^{-4}$ & $7.28868^{-4}$ & $8.82835^{-4}$ \\
\hline
\end{tabular}

TABLE 8: Fourth-order absolute residual errors $|\operatorname{Re}|$ for different values of $\eta$ and $\alpha$, at $R=0.3, M=0.7$, and $\gamma=1$ in case of slip boundary, by the two methods.

\begin{tabular}{lcccccc}
\hline$\eta$ & \multicolumn{1}{c}{ PM } & & & \multicolumn{2}{c}{ ADM } \\
& $\alpha=3.9$ & $\alpha=3.95$ & $\alpha=4.0$ & $\alpha=4.0$ & $\alpha=3.95$ & 0.0 \\
\hline 0.0 & 0.0 & 0.0 & 0.0 & 0.0 & $8.28814^{-9}$ & $1.20954^{-8}$ \\
0.1 & $1.76581^{-8}$ & $1.21259^{-8}$ & $8.30938^{-9}$ & $2.54571^{-7}$ & $3.46670^{-7}$ & $1.76144^{-8}$ \\
0.2 & $4.75929^{-7}$ & $3.50290^{-7}$ & $2.57276^{-7}$ & $4.71090^{-7}$ \\
0.3 & $3.15104^{-6}$ & $2.41525^{-6}$ & $1.84736^{-6}$ & $1.80153^{-6}$ & $2.35635^{-6}$ & $3.07545^{-6}$ \\
0.4 & $1.15658^{-5}$ & $9.12382^{-6}$ & $7.18218^{-6}$ & $6.84284^{-6}$ & $8.70018^{-6}$ & $1.10377^{-5}$ \\
0.5 & $3.03150^{-5}$ & $2.44516^{-5}$ & $1.96804^{-5}$ & $1.80871^{-5}$ & $2.25070^{-5}$ & $2.79450^{-5}$ \\
0.6 & $6.33559^{-5}$ & $5.20277^{-5}$ & $4.26341^{-5}$ & $3.70338^{-5}$ & $4.53191^{-5}$ & $5.53316^{-5}$ \\
0.7 & $1.11599^{-4}$ & $9.30102^{-5}$ & $7.73532^{-5}$ & $6.12585^{-5}$ & $7.40371^{-5}$ & $8.92657^{-5}$ \\
0.8 & $1.70455^{-4}$ & $1.43790^{-4}$ & $1.21040^{-4}$ & $8.11811^{-5}$ & $9.74626^{-5}$ & $1.16691^{-4}$ \\
0.9 & $2.28293^{-4}$ & $1.94372^{-4}$ & $1.65144^{-4}$ & $7.71638^{-5}$ & $9.34133^{-5}$ & $1.12625^{-4}$ \\
1.0 & $2.66966^{-4}$ & $2.28522^{-4}$ & $1.95203^{-4}$ & $1.81316^{-5}$ & $2.77087^{-5}$
\end{tabular}

other parameters fixed for different order solutions obtained by the two methods in case of no-slip and slip boundaries.

For various values of $R$ and $M$, the results in Tables 1 , 2, 5, and 6 indicate that as $\alpha \rightarrow 4, u_{\mathrm{ADM}} \rightarrow u_{\mathrm{PM}}$ and the results in Tables 3, 4, 7, and 8 (for 4th-order solution), 9 and 10 (for 3 rd-order solution), and 11 and 12 (for 2 nd-order solution) indicate that increasing $\alpha$ and converging it to 4 implies decreasing the absolute residual errors $|\operatorname{Re}|$ as $M \rightarrow 1$. 
TABLE 9: Third-order absolute residual errors $|\operatorname{Re}|$ for different values of $\eta$ and $\alpha$, at $R=0.3, M=0.5, \gamma=1$ by the two methods.

\begin{tabular}{lcccccc}
\hline$\eta$ & & No-slip & & \multicolumn{2}{c}{ Slip } \\
& $\alpha=3.9$ & $\alpha=3.95$ & $\alpha=4.0$ & $\alpha=4.0$ & $\alpha=3.95$ & 0.0 \\
0.0 & 0.0 & 0.0 & 0.0 & 0.0 & $7.38815^{-5}$ & $8.77102^{-5}$ \\
0.1 & $5.48712^{-6}$ & $5.70765^{-6}$ & $5.70833^{-6}$ & $5.82986^{-4}$ & $6.68560^{-4}$ & $7.04048^{-4}$ \\
0.2 & $2.39714^{-5}$ & $2.91487^{-5}$ & $3.25070^{-5}$ & $1.92251^{-3}$ & $2.16064^{-3}$ & $2.42644^{-3}$ \\
0.3 & $1.63919^{-5}$ & $1.19104^{-5}$ & $3.38931^{-5}$ & $4.40898^{-3}$ & $4.88503^{-3}$ & $5.40837^{-3}$ \\
0.4 & $3.52371^{-4}$ & $2.58577^{-3}$ & $1.80174^{-4}$ & $8.24444^{-3}$ & $9.03521^{-3}$ & $9.89430^{-3}$ \\
0.5 & $1.48243^{-3}$ & $1.24374^{-3}$ & $1.03582^{-3}$ & $1.34847^{-2}$ & $1.46487^{-2}$ & $1.59010^{-2}$ \\
0.6 & $4.28883^{-3}$ & $3.77352^{-3}$ & $3.31245^{-3}$ & $2.15836^{-2}$ & $2.32596^{-2}$ \\
0.7 & $1.01929^{-2}$ & $9.19883^{-3}$ & $8.29226^{-3}$ & $2.00130^{-2}$ & $2.95023^{-2}$ & $3.16030^{-2}$ \\
0.8 & $2.13503^{-2}$ & $1.95839^{-2}$ & $1.79494^{-2}$ & $2.75203^{-2}$ & $3.78627^{-2}$ & $4.03598^{-2}$ \\
0.9 & $4.08930^{-2}$ & $3.79446^{-2}$ & $3.51850^{-2}$ & $3.54931^{-2}$ & $4.59178^{-2}$ \\
1.0 & $7.32218^{-2}$ & $6.85388^{-2}$ & $6.41147^{-2}$ & $4.32097^{-2}$ & $4.87584^{-2}$ \\
\hline
\end{tabular}

TABLE 10: Third-order absolute residual errors $\mid$ Re $\mid$ for different values of $\eta$ and $\alpha$, at $R=0.3, M=0.7$, and $\gamma=1$ by the two methods.

\begin{tabular}{|c|c|c|c|c|c|c|}
\hline \multirow{2}{*}{$\eta$} & \multicolumn{3}{|c|}{ No-slip } & \multicolumn{3}{|c|}{ Slip } \\
\hline & $\alpha=3.9$ & $\alpha=3.95$ & $\alpha=4.0$ & $\alpha=4.0$ & $\alpha=3.95$ & $\alpha=3.9$ \\
\hline 0.0 & 0.0 & 0.0 & 0.0 & 0.0 & 0.0 & 0.0 \\
\hline 0.1 & $8.37934^{-6}$ & $7.24173^{-6}$ & $6.24827^{-6}$ & $2.90165^{-5}$ & $3.43492^{-5}$ & $4.06294^{-5}$ \\
\hline 0.2 & $6.24359^{-5}$ & $5.57928^{-5}$ & $4.97782^{-5}$ & $2.27300^{-4}$ & $2.59907^{-4}$ & $2.96952^{-4}$ \\
\hline 0.3 & $1.99303^{-4}$ & $1.81455^{-4}$ & $1.64961^{-4}$ & $7.40219^{-4}$ & $8.29420^{-4}$ & $9.28619^{-4}$ \\
\hline 0.4 & $4.37350^{-4}$ & $4.03436^{-4}$ & $3.71623^{-4}$ & $1.66653^{-3}$ & $1.84074^{-3}$ & $2.03150^{-3}$ \\
\hline 0.5 & $7.43336^{-4}$ & $6.93453^{-4}$ & $6.45982^{-4}$ & $3.03807^{-3}$ & $3.31867^{-3}$ & $3.62221^{-3}$ \\
\hline 0.6 & $9.70033^{-4}$ & $9.17089^{-4}$ & $8.65511^{-4}$ & $4.80293^{-3}$ & $5.19979^{-3}$ & $5.62479^{-3}$ \\
\hline 0.7 & $7.56286^{-4}$ & $7.39037^{-4}$ & $7.19177^{-4}$ & $6.81333^{-3}$ & $7.32212^{-3}$ & $7.86235^{-3}$ \\
\hline 0.8 & $6.18418^{-4}$ & $5.15911^{-4}$ & $4.25325^{-4}$ & $8.81918^{-3}$ & $9.42063^{-3}$ & $1.00546^{-2}$ \\
\hline 0.9 & $4.43231^{-3}$ & $4.05396^{-3}$ & $3.70548^{-3}$ & $1.04684^{-2}$ & $1.11297^{-2}$ & $1.18228^{-2}$ \\
\hline 1.0 & $1.27795^{-2}$ & $1.18628^{-2}$ & $1.10067^{-2}$ & $1.13149^{-2}$ & $1.19937^{-2}$ & $1.27021^{-2}$ \\
\hline
\end{tabular}

TABLE 11: Second-order absolute residual errors $|\operatorname{Re}|$ for different values of $\eta$ and $\alpha$, at $R=0.3, M=0.5$, and $\gamma=1$ by the two methods.

\begin{tabular}{|c|c|c|c|c|c|c|}
\hline \multirow{2}{*}{$\eta$} & \multicolumn{3}{|c|}{ No-slip } & \multicolumn{3}{|c|}{ Slip } \\
\hline & $\alpha=3.9$ & $\alpha=3.95$ & $\alpha=4.0$ & $\alpha=4.0$ & $\alpha=3.95$ & $\alpha=3.9$ \\
\hline 0.0 & 0.0 & 0.0 & 0.0 & 0.0 & 0.0 & 0.0 \\
\hline 0.1 & $4.48712^{-5}$ & $4.70765^{-5}$ & $4.70833^{-5}$ & $6.38815^{-4}$ & $7.77102^{-4}$ & $1.02048^{-3}$ \\
\hline 0.2 & $1.39714^{-4}$ & $3.91487^{-4}$ & $2.25070^{-4}$ & $4.82986^{-3}$ & $5.68560^{-3}$ & $6.66115^{-3}$ \\
\hline 0.3 & $2.63919^{-4}$ & $2.19104^{-4}$ & $2.38931^{-4}$ & $1.72251^{-2}$ & $2.11064^{-2}$ & $2.42644^{-2}$ \\
\hline 0.4 & $3.52371^{-3}$ & $3.58577^{-2}$ & $1.80174^{-3}$ & $4.20898^{-2}$ & $4.77503^{-2}$ & $5.50837^{-2}$ \\
\hline 0.5 & $2.48243^{-2}$ & $1.24374^{-2}$ & $2.03582^{-2}$ & $7.24444^{-2}$ & $8.03521^{-2}$ & $8.89430^{-2}$ \\
\hline 0.6 & $4.28883^{-2}$ & $3.87352^{-2}$ & $3.41245^{-2}$ & $1.14847^{-1}$ & $1.12487^{-1}$ & $1.69010^{-1}$ \\
\hline 0.7 & $2.01929^{-1}$ & $8.19883^{-2}$ & $7.29226^{-2}$ & $1.01130^{-1}$ & $2.05836^{-1}$ & $2.42596^{-1}$ \\
\hline 0.8 & $2.13503^{-1}$ & $1.85839^{-1}$ & $1.89494^{-1}$ & $2.65203^{-1}$ & $2.75023^{-1}$ & $3.12030^{-1}$ \\
\hline 0.9 & $3.08930^{-1}$ & $3.99446^{-1}$ & $3.71850^{-1}$ & $3.44931^{-1}$ & $3.58627^{-1}$ & $4.13598^{-1}$ \\
\hline 1.0 & $6.32218^{-1}$ & $5.85388^{-1}$ & $6.71147^{-1}$ & $3.32097^{-1}$ & $4.39178^{-1}$ & $4.77584^{-1}$ \\
\hline
\end{tabular}

Also, increasing the order of solution implies decreasing $|\mathrm{Re}|$. Moreover, the results in Tables 3 and 4 indicate that $|\mathrm{Re}|$ obtained by PM are less than $|\operatorname{Re}|$ that obtained by ADM in case of no-slip boundary, while, in case of slip boundary, the results in Tables 7 and 8 indicate the inverse. In addition to the abovementioned, we noted from the results in Tables 13 and 14 that the absolute difference between the ADM and PM solutions are almost equal especially for small values of $\eta$ for different values of $\alpha, M$, and $R$ at $\gamma=1$ which means that the two methods are in a good agreement in solving the proposed problem and therefore the ADM is a reliable method in solving the fractional order problems Finally, the results in 
TABLE 12: Second-order absolute residual errors $|\operatorname{Re}|$ for different values of $\eta$ and $\alpha$, at $R=0.3, M=0.7$, and $\gamma=1$ by the two methods.

\begin{tabular}{|c|c|c|c|c|c|c|}
\hline \multirow{2}{*}{$\eta$} & \multicolumn{3}{|c|}{ No-slip } & \multicolumn{3}{|c|}{ Slip } \\
\hline & $\alpha=3.9$ & $\alpha=3.95$ & $\alpha=4.0$ & $\alpha=4.0$ & $\alpha=3.95$ & $\alpha=3.9$ \\
\hline 0.0 & 0.0 & 0.0 & 0.0 & 0.0 & 0.0 & 0.0 \\
\hline 0.1 & $7.37934^{-5}$ & $6.24173^{-5}$ & $5.24827^{-5}$ & $1.90165^{-4}$ & $2.43492^{-4}$ & $3.06294^{-4}$ \\
\hline 0.2 & $5.24359^{-4}$ & $5.47928^{-4}$ & $4.77782^{-4}$ & $1.27300^{-3}$ & $1.59907^{-3}$ & $1.96952^{-3}$ \\
\hline 0.3 & $1.77303^{-3}$ & $1.61455^{-3}$ & $1.44961^{-3}$ & $6.40219^{-3}$ & $7.29420^{-3}$ & $8.28619^{-3}$ \\
\hline 0.4 & $4.32350^{-3}$ & $4.01436^{-3}$ & $3.61623^{-3}$ & $1.55653^{-2}$ & $1.64074^{-2}$ & $1.93150^{-2}$ \\
\hline 0.5 & $6.43336^{-3}$ & $6.63453^{-3}$ & $6.45982^{-3}$ & $2.03807^{-2}$ & $3.31867^{-2}$ & $3.63221^{-2}$ \\
\hline 0.6 & $8.70033^{-3}$ & $8.17089^{-3}$ & $8.15511^{-3}$ & $3.80293^{-2}$ & $5.29979^{-2}$ & $5.52479^{-2}$ \\
\hline 0.7 & $6.56286^{-3}$ & $6.39037^{-3}$ & $6.19177^{-3}$ & $5.81333^{-2}$ & $7.11212^{-2}$ & $7.66235^{-2}$ \\
\hline 0.8 & $5.18418^{-3}$ & $5.05911^{-3}$ & $4.35325^{-3}$ & $7.81918^{-2}$ & $8.42063^{-2}$ & $1.00546^{-1}$ \\
\hline 0.9 & $4.23231^{-2}$ & $4.01396^{-2}$ & $3.71548^{-2}$ & $1.01684^{-1}$ & $1.11297^{-1}$ & $1.17228^{-1}$ \\
\hline 1.0 & $1.17795^{-1}$ & $1.15828^{-1}$ & $1.11067^{-1}$ & $1.12149^{-1}$ & $1.18837^{-1}$ & $1.25021^{-1}$ \\
\hline
\end{tabular}

TABLE 13: Absolute difference of ADM and PM solutions; $\left|u_{\mathrm{ADM}}-u_{\mathrm{PM}}\right|$ for various values of $\eta$ and $\alpha$, at $R=0.3, M=0.5$, and $\gamma=1$.

\begin{tabular}{|c|c|c|c|c|c|c|}
\hline \multirow{2}{*}{$\eta$} & \multicolumn{3}{|c|}{ No-slip } & \multicolumn{3}{|c|}{ Slip } \\
\hline & $\alpha=3.9$ & $\alpha=3.95$ & $\alpha=4.0$ & $\alpha=4.0$ & $\alpha=3.95$ & $\alpha=3.9$ \\
\hline 0.0 & 0.0 & 0.0 & 0.0 & 0.0 & 0.0 & 0.0 \\
\hline 0.1 & $2.77556^{-17}$ & $2.77556^{-17}$ & 0.0 & 0.0 & $1.38778^{-17}$ & $1.38778^{-17}$ \\
\hline 0.2 & $5.44009^{-15}$ & $3.10862^{-15}$ & $1.83187^{-15}$ & $1.63758^{-15}$ & $2.83107^{-15}$ & $4.77396^{-15}$ \\
\hline 0.3 & $4.13447^{-13}$ & $2.57239^{-13}$ & $1.59817^{-13}$ & $1.41387^{-13}$ & $2.27457^{-13}$ & $3.65152^{-13}$ \\
\hline 0.4 & $9.09539^{-12}$ & $5.90838^{-12}$ & $3.83005^{-12}$ & $3.33550^{-12}$ & $5.14022^{-12}$ & $7.90479^{-12}$ \\
\hline 0.5 & $1.00677^{-10}$ & $6.75984^{-11}$ & $4.52951^{-11}$ & $3.86652^{-11}$ & $5.76179^{-11}$ & $8.56799^{-11}$ \\
\hline 0.6 & $7.22511^{-10}$ & $4.98311^{-10}$ & $3.42987^{-10}$ & $2.85796^{-10}$ & $4.14338^{-10}$ & $5.99424^{-10}$ \\
\hline 0.7 & $3.84849^{-9}$ & $2.71476^{-9}$ & $1.91119^{-9}$ & $1.54808^{-9}$ & $2.19269^{-9}$ & $3.09911^{-9}$ \\
\hline 0.8 & $1.64931^{-8}$ & $1.18619^{-8}$ & $8.51429^{-9}$ & $6.67733^{-9}$ & $9.26833^{-9}$ & $1.28373^{-8}$ \\
\hline 0.9 & $5.99027^{-8}$ & $4.38185^{-8}$ & $3.19905^{-8}$ & $2.41963^{-8}$ & $3.29895^{-8}$ & $4.48821^{-8}$ \\
\hline 1.0 & $9.91033^{-8}$ & $9.41855^{-8}$ & $9.05135^{-8}$ & $7.64034^{-8}$ & $9.02510^{-8}$ & $9.37241^{-8}$ \\
\hline
\end{tabular}

TABLE 14: Absolute difference of ADM and PM solutions; $\left|u_{\mathrm{ADM}}-u_{\mathrm{PM}}\right|$ for various values of $\eta$ and $\alpha$, at $R=0.3, M=0.7$, and $\gamma=1$.

\begin{tabular}{lcccccc}
\hline$\eta$ & & No-slip & & \multicolumn{2}{c}{ Slip } \\
$\alpha=3.95$ & $\alpha=4.0$ & 0.0 & $\alpha=3.9$ \\
\hline 0.0 & $\alpha=3.9$ & $\alpha=3.95$ & 0.0 & 0.0 & 0.0 & $1.38778^{-17}$ \\
0.1 & $5.55112^{-17}$ & $2.77556^{-17}$ & 0.0 & $2.77556^{-17}$ & $1.38778^{-17}$ \\
0.2 & $2.77556^{-16}$ & $5.55112^{-17}$ & $5.55112^{-17}$ & $5.55112^{-16}$ & $9.15934^{-16}$ & $1.58207^{-15}$ \\
0.3 & $1.77081^{-14}$ & $1.09357^{-14}$ & $6.77236^{-15}$ & $4.72677^{-14}$ & $7.59393^{-14}$ & $1.22014^{-13}$ \\
0.4 & $4.07563^{-13}$ & $2.64122^{-13}$ & $1.70641^{-13}$ & $1.11194^{-12}$ & $1.71324^{-12}$ & $2.63423^{-12}$ \\
0.5 & $4.78895^{-12}$ & $3.20177^{-12}$ & $2.13662^{-12}$ & $1.28488^{-11}$ & $1.91425^{-11}$ & $2.84585^{-11}$ \\
0.6 & $3.67931^{-11}$ & $2.52315^{-11}$ & $1.72713^{-11}$ & $9.46089^{-11}$ & $1.37114^{-10}$ & $1.98292^{-10}$ \\
0.7 & $2.11315^{-10}$ & $1.47993^{-10}$ & $1.03464^{-10}$ & $5.10129^{-10}$ & $7.22201^{-10}$ & $1.02024^{-9}$ \\
0.8 & $9.81739^{-10}$ & $6.99977^{-10}$ & $4.98231^{-10}$ & $2.18861^{-9}$ & $3.03596^{-9}$ & $4.20227^{-9}$ \\
0.9 & $3.88008^{-9}$ & $2.80987^{-7}$ & $2.03147^{-9}$ & $7.88225^{-9}$ & $1.07381^{-8}$ & $1.45969^{-8}$ \\
1.0 & $1.34971^{-8}$ & $9.90959^{-9}$ & $7.26394^{-9}$ & $2.47168^{-8}$ & $3.31290^{-8}$ & $4.43064^{-8}$ \\
\hline
\end{tabular}

Tables 9 and 10 and 11 and 12 indicate that the $3 r d$-order and 2nd-order absolute residual errors $|\mathrm{Re}|$, respectively, are equal for the two methods in case of no-slip and slip boundaries.

Figures 1 and 2 show the residual errors Re for various values of $\alpha, M$, and $R$ by PM in cases of no-slip and slip boundaries with $\gamma=1$ (Figure 1), while in Figure 2 it is by ADM.

The effect of different values of the Reynolds number $R$ and the constant containing permeability $M$ at the fractional order $\alpha=3.7$ on velocity profiles of the two methods in case 
TABLE 15: Average absolute residual errors for fourth-order solutions for various values of $\alpha$, at $R=0.3, M=0.7$, and $\gamma=1$ by the two methods.

\begin{tabular}{|c|c|c|c|c|c|}
\hline \multirow{2}{*}{$\alpha$} & \multirow{2}{*}{$|\operatorname{Re}|$} & \multicolumn{2}{|c|}{ No-slip } & \multicolumn{2}{|c|}{ Slip } \\
\hline & & PM & $\mathrm{ADM}$ & $\mathrm{PM}$ & $\operatorname{ADM} \alpha=3.9$ \\
\hline \multirow{3}{*}{$\alpha=3.9$} & Minimum & 0.0 & 0.0 & 0.0 & 0.0 \\
\hline & Average & $8.21978^{-5}$ & $2.83204^{-4}$ & $8.05631^{-5}$ & $4.14605^{-5}$ \\
\hline & Maximum & $2.64276^{-5}$ & $1.15284^{-4}$ & $2.66966^{-4}$ & $1.16691^{-4}$ \\
\hline \multirow{3}{*}{$\alpha=3.95$} & Minimum & 0.0 & 0.0 & 0.0 & 0.0 \\
\hline & Average & $6.69461^{-5}$ & $2.40512^{-4}$ & $6.80068^{-5}$ & $3.38057^{-5}$ \\
\hline & Maximum & $2.20234^{-5}$ & $9.68709^{-5}$ & $2.28522^{-4}$ & $9.34133^{-5}$ \\
\hline \multirow{3}{*}{$\alpha=4.0$} & Minimum & 0.0 & 0.0 & 0.0 & 0.0 \\
\hline & Average & $4.99631^{-6}$ & $1.23248^{-5}$ & $5.73045^{-5}$ & $2.74330^{-5}$ \\
\hline & Maximum & $1.92411^{-5}$ & $8.14057^{-5}$ & $1.95203^{-4}$ & $1.81316^{-5}$ \\
\hline
\end{tabular}

TABLE 16: Average absolute residual errors for third-order and second-order solutions for various values of $\alpha$, at $R=0.3, M=0.7$, and $\gamma=1$ by the two methods.

\begin{tabular}{|c|c|c|c|c|c|}
\hline \multirow{2}{*}{$\alpha$} & \multirow{2}{*}{$|\operatorname{Re}|$} & \multicolumn{2}{|c|}{ Third-order } & \multicolumn{2}{|c|}{ Second-order } \\
\hline & & No-slip & Slip & No-slip & Slip \\
\hline \multirow{3}{*}{$\alpha=3.9$} & Minimum & 0.0 & 0.0 & 0.0 & 0.0 \\
\hline & Average & $1.90976^{-3}$ & $4.99878^{-3}$ & $1.76085^{-2}$ & $4.91696^{-2}$ \\
\hline & Maximum & $1.27795^{-2}$ & $1.27021^{-2}$ & $1.17795^{-1}$ & $1.25021^{-1}$ \\
\hline \multirow{3}{*}{$\alpha=3.95$} & Minimum & 0.0 & 0.0 & 0.0 & 0.0 \\
\hline & Average & $1.76729^{-3}$ & $4.66809^{-3}$ & $1.74056^{-2}$ & $4.51991^{-2}$ \\
\hline & Maximum & $1.18628^{-2}$ & $1.19937^{-2}$ & $1.15828^{-1}$ & $1.18837^{-1}$ \\
\hline \multirow{3}{*}{$\alpha=4.0$} & Minimum & 0.0 & 0.0 & 0.0 & 0.0 \\
\hline & Average & $1.63280^{-3}$ & $3.79890^{-3}$ & $1.62707^{-2}$ & $3.92726^{-2}$ \\
\hline & Maximum & $1.10067^{-2}$ & $1.13149^{-2}$ & $1.11067^{-1}$ & $1.12149^{-1}$ \\
\hline
\end{tabular}

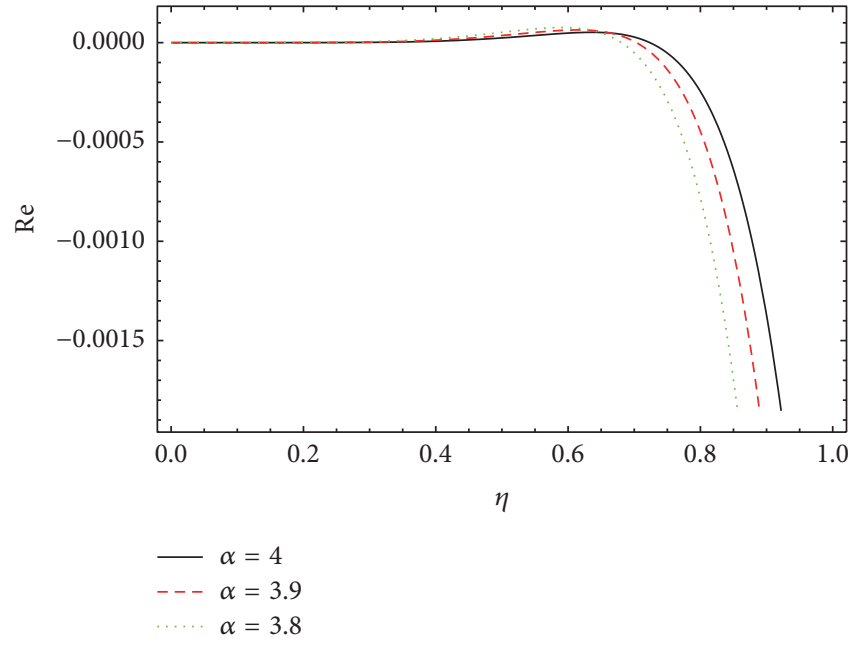

(a)

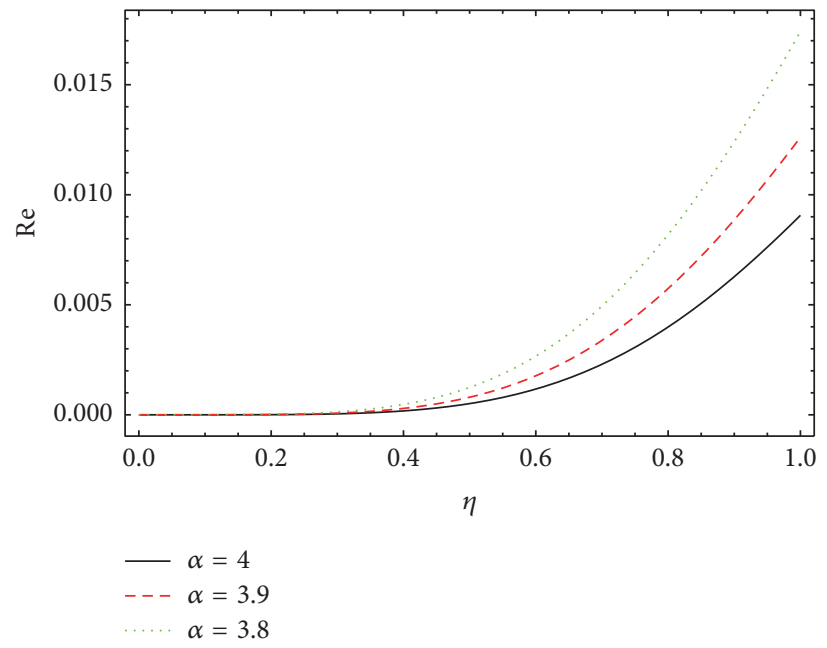

(b)

FiguRE 1: Residuals for various values of $\alpha$ at $R=.5, M=.7$, and $\gamma=1$ by PM: (a) in case of no-slip boundary and (b) in case of slip boundary.

of no-slip boundary is shown in Figures 3 and 4, while the effect in case of slip boundary for different values of $R, M$, and slip parameter $\gamma$ at $\alpha=3.7$ are shown in Figures 5, 6, and 7. In these profiles we varied $R$ as $R=1,2,3$ for the sake of comparison at $M=3$ and $\alpha=3.7$ (Figure 3) and varied $M$ as $M=1,2,3$ for the sake of comparison at $R=3$ and $\alpha=3.7$ (Figure 4 ) in case of no-slip boundary. In case of slip boundary, we varied $R$ as $R=1,2,3$ for the sake of 


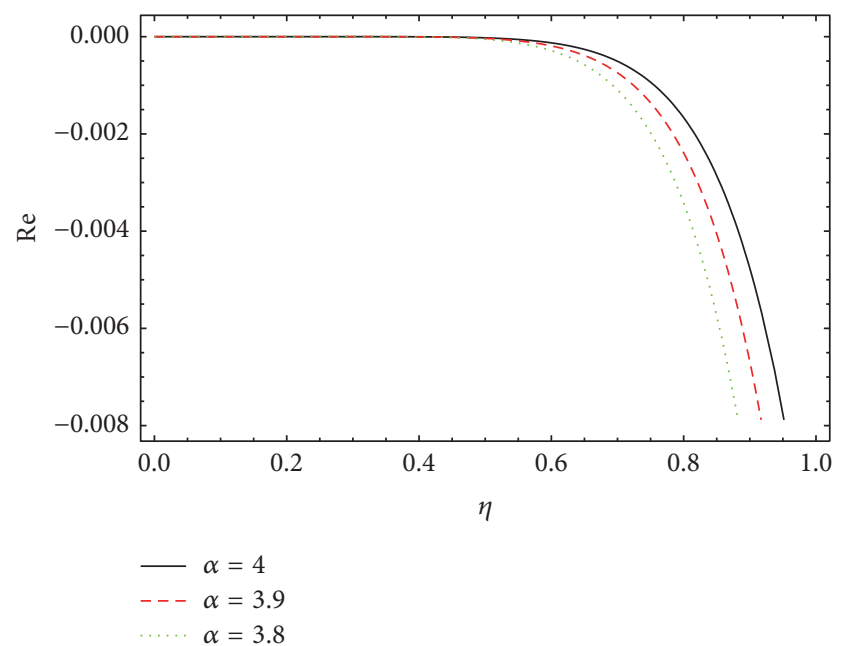

(a)

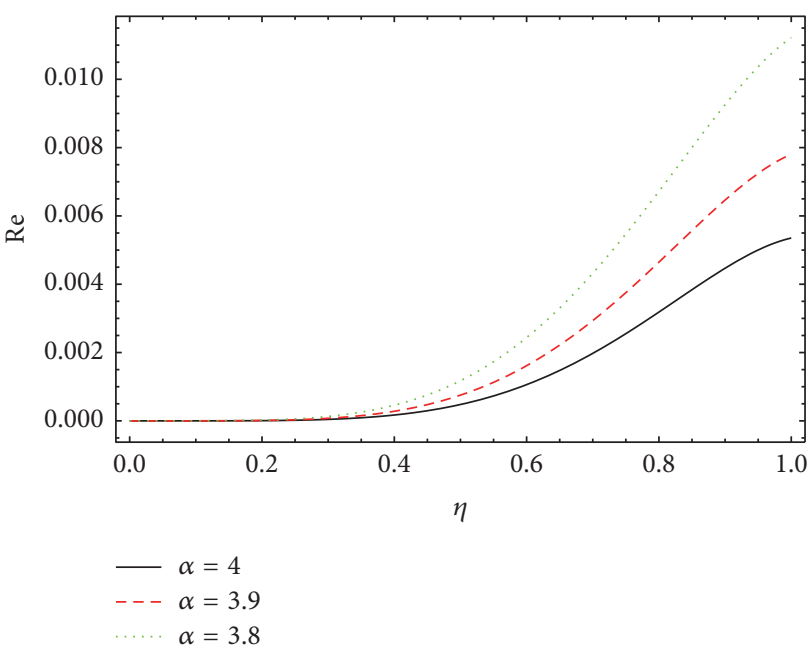

(b)

Figure 2: Residuals for various values of $\alpha$ at $R=.5, M=.7$, and $\gamma=1$ by ADM: (a) in case of no-slip boundary and (b) in case of slip boundary.

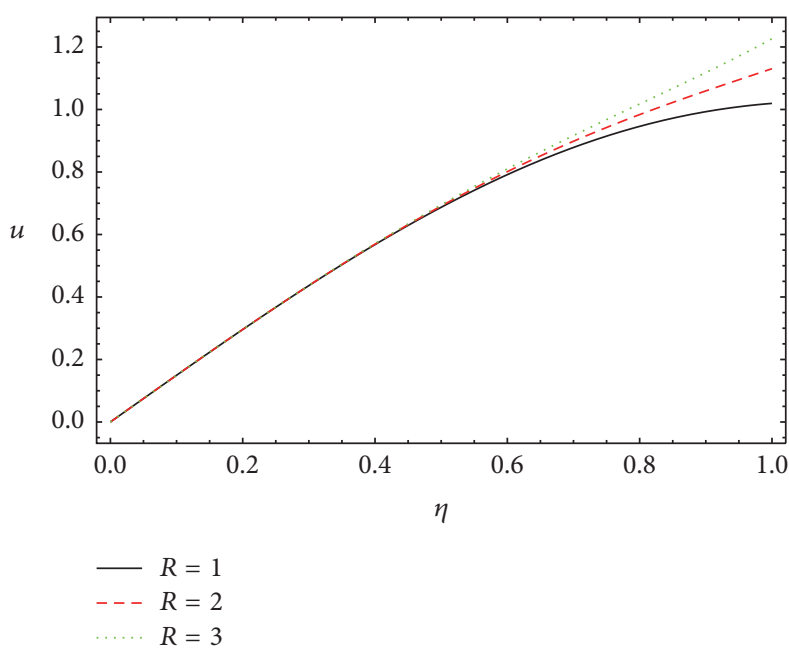

(a)

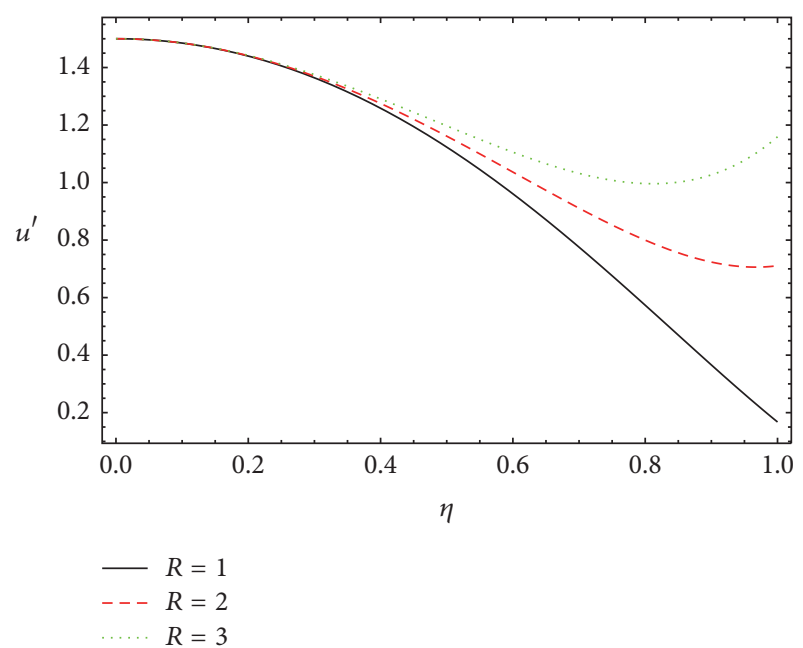

(b)

FIGURE 3: Velocity profiles for various values of $R$ at $\alpha=3.7$ and $M=3$ by PM in case of no-slip boundary: (a) normal velocity and (b) longitudinal velocity.

comparison at $M=3, \gamma=1$, and $\alpha=3.7$ (Figure 5), varied $M$ as $M=1,2,3$ for the sake of comparison at $R=3, \gamma=1$, and $\alpha=3.7$ (Figure 6), and varied $\gamma$ as $\gamma=.75,1,1.25$ for the sake of comparison at $R=M=3$ and $\alpha=3.7$ (Figure 7).

According to the abovementioned data we have observed that, in case of no-slip boundary, the normal velocity (NV) increases with increasing $R$ (Figure 3(a)) and the longitudinal velocity (LV) increases near the central axis of the channel (Figure 3(b)). It has been analyzed that the NV monotonically increases while the LV monotonically decreases from $\eta=$ 0 to $\eta=1$ for fixed value of $R$ at a given time. Almost a similar behavior for the NV and LV is with varying $M$ (Figure 4). In case of slip boundary, we have noted that the NV decreases as $R$ increases (Figure 5(a)) and the LV decreases near the central axis of the channel (Figure 5(b)). Also, almost a similar behavior for the NV and LV is with varying $M$ (Figure 6). Moreover, we have observed that the $\mathrm{NV}$ increases with increasing $\gamma$ (Figure $7(\mathrm{a})$ ) and the LV increases near the wall and decreases near the central axis of the channel (Figure 7(b)).

\section{Conclusion}

In this work, we find the analytical solution and then the similarity solution for the fractional form of an unsteady axisymmetric flow of incompressible, nonconducting Newtonian fluid squeezed between two circular plates passing through porous medium channel using the PM and ADM 


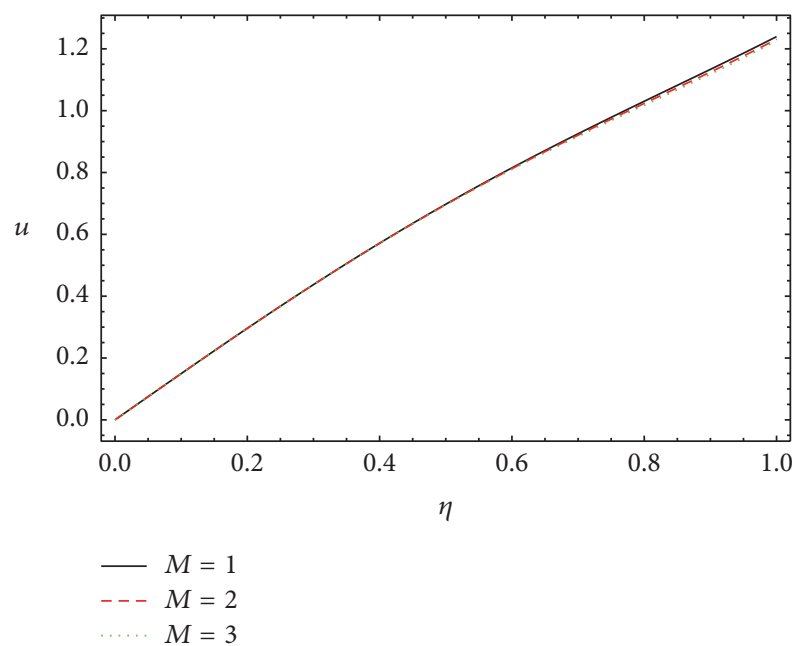

(a)

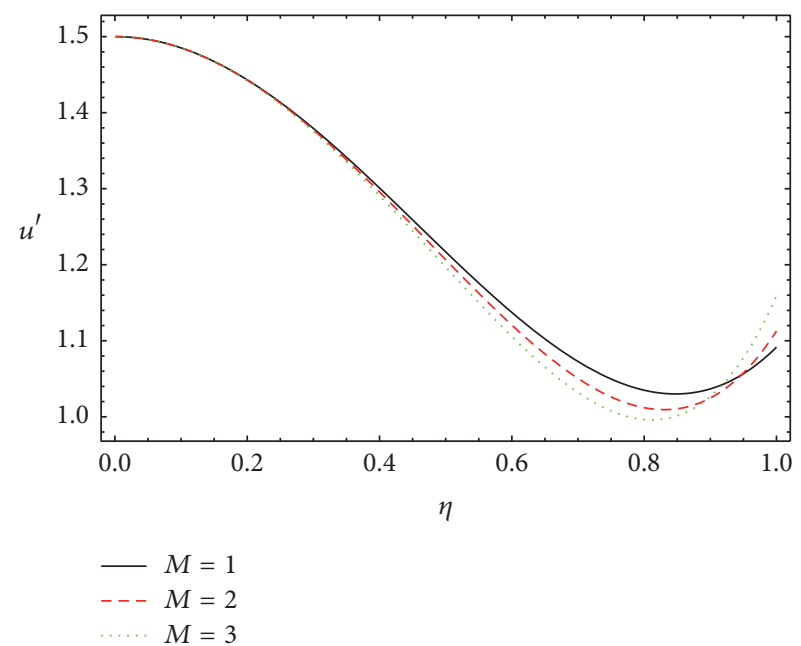

(b)

FIgURE 4: Velocity profiles for various values of $M$ at $\alpha=3.7$ and $R=3$ by ADM in case of no-slip boundary: (a) normal velocity and (b) longitudinal velocity.

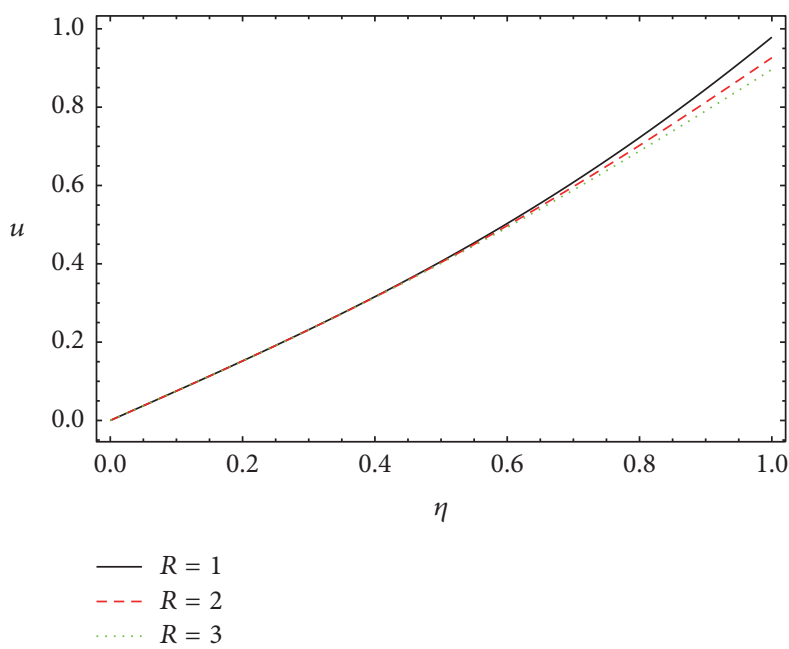

(a)

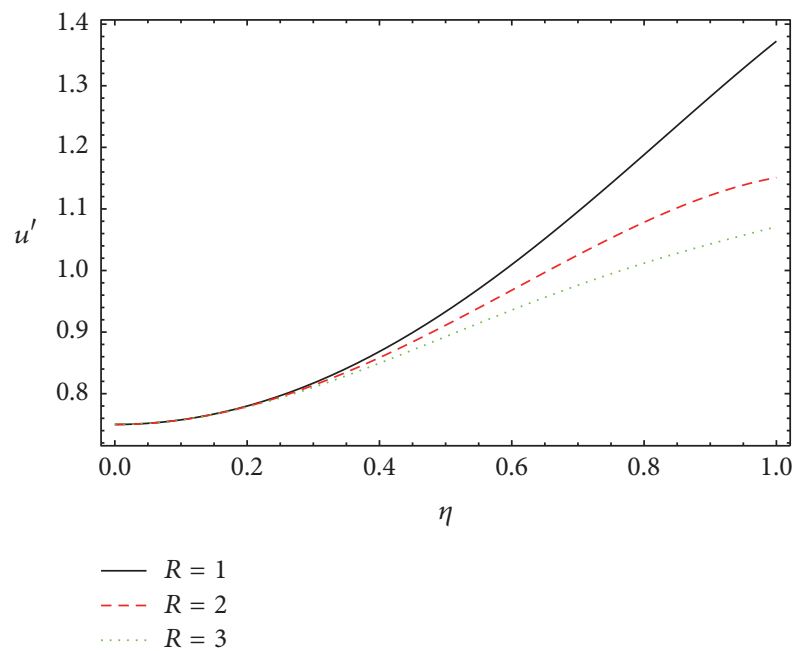

(b)

Figure 5: Velocity profiles for various values of $R$ at $\alpha=3.7, M=3$, and $\gamma=1$ by PM in case of slip boundary: (a) normal velocity and (b) longitudinal velocity.

in cases of no-slip and slip boundary conditions. The convergence of the ADM is determined through various order approximate solutions. Moreover, the validity of ADM is checked by comparing the analytical ADM and the numerical PM solutions. We observed that the similarity solution occurs when the distance between the plates varies as $(c t+d)^{1 / 2}$ and squeezing flow exists when $c<0, d>0$ and $(c t+d)^{1 / 2}>0$. Some key findings related to the present study are as follows.

In Case of No-Slip Boundary. (i) The NV increases with the increase in $R$. (ii) The LV increases near the central axis of the channel with the increase in $R$. (iii) The NV increases monotonically while the LV decreases monotonically from $\eta=0$ to $\eta=1$ for fixed value of $R$ at a given time. (iv) For the variation in $M$, we have noted almost a similar behavior.

In Case of Slip Boundary. (i) The NV decreases with the increase in $R$ with fixed $M$ and $\gamma$. (ii) The LV decreases near the central axis of the channel with the increase in $R$ with fixed $M$ and $\gamma$. (iii) For the variation in $M$, we have observed almost a similar behavior for fixed $R$ and $\gamma$. (iv) The NV increases with the increase in $\gamma$ with fixed $R$ and $M$. (v) The $\mathrm{LV}$ increases near the wall and decreases near the central axis of the channel with fixed $R$ and $M$. (vi) $R$ and $M$ have similar effects on the NV and LV, while $R, \gamma$ have opposite effects on the NV and LV components. 


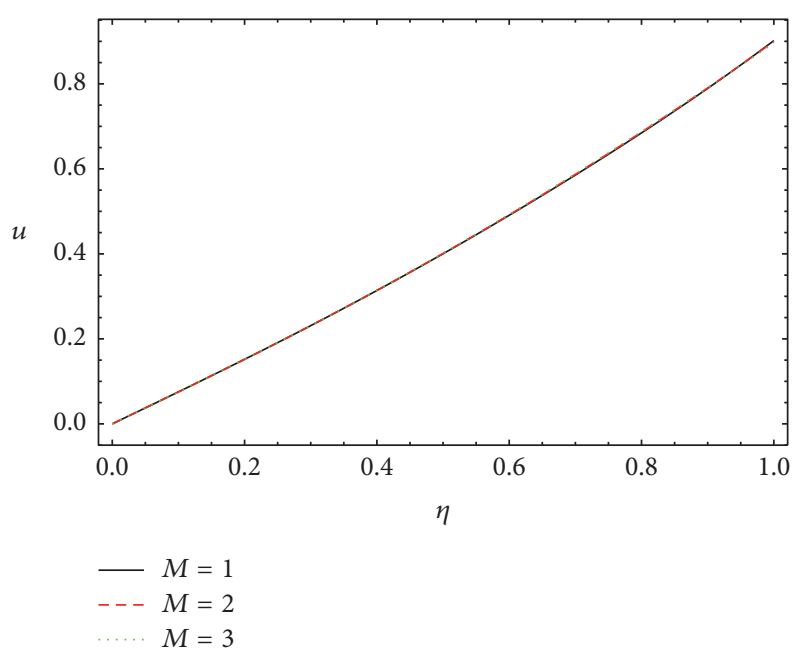

(a)

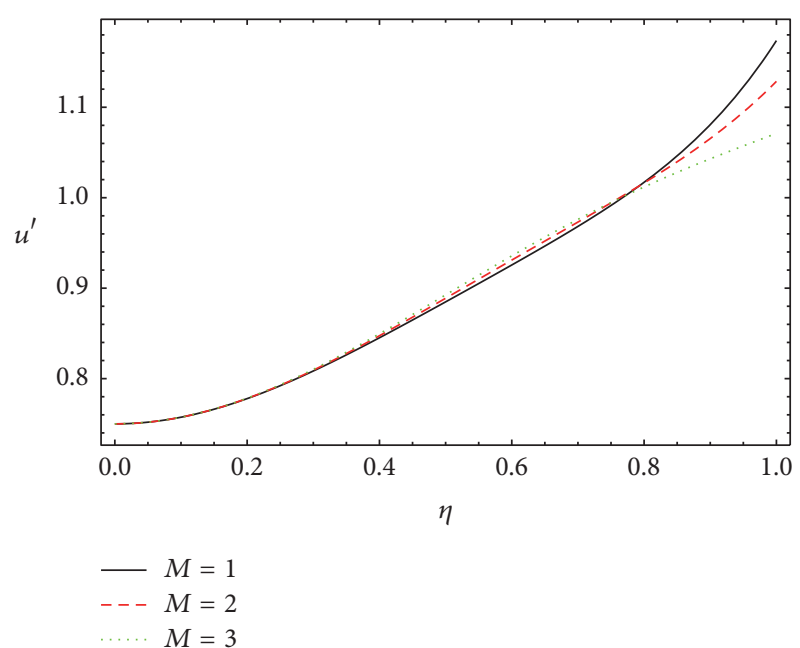

(b)

Figure 6: Velocity profiles for various values of $M$ at $\alpha=3.7, R=3$, and $\gamma=1$ by ADM in case of slip boundary: (a) normal velocity and (b) longitudinal velocity.

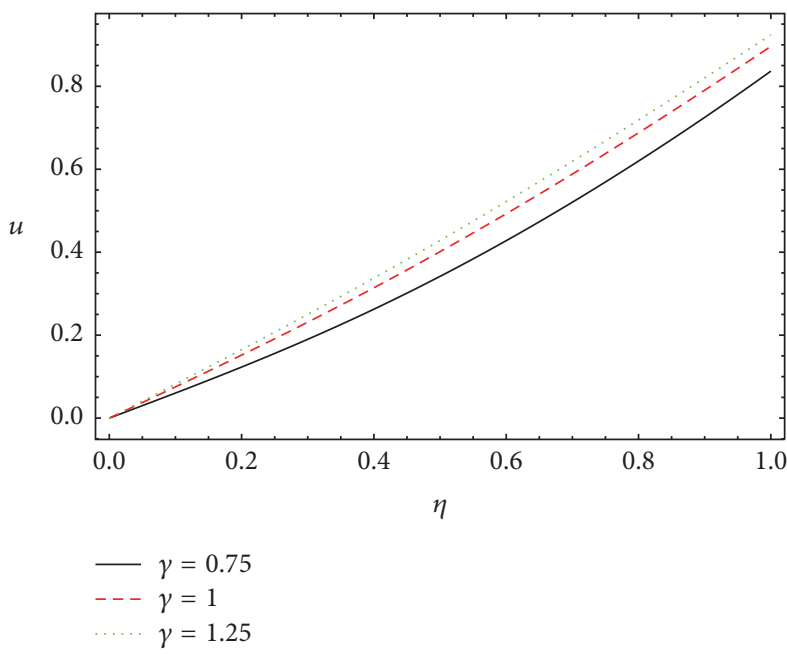

(a)

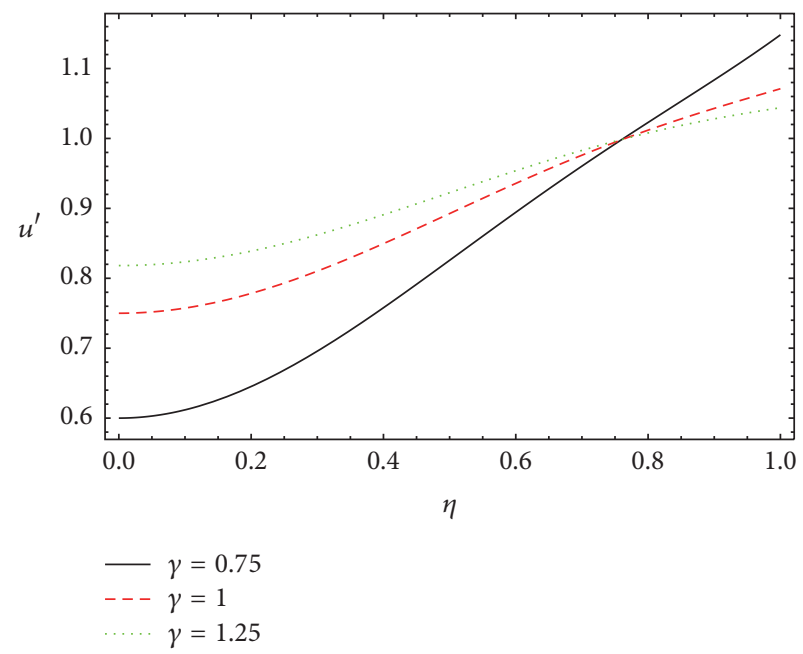

(b)

FIGURE 7: Velocity profiles for various values of $\gamma$ at $\alpha=3.7, R=M=3$ by ADM in case of slip boundary: (a) normal velocity and (b) longitudinal velocity.

In Both Cases of No-Slip and Slip Boundaries. (i) It has been found that the changes in velocity profiles with varying $\alpha$ are less for small values of $R$ and $M$. (ii) It has been found that the residual errors in the $3 r d$-order solution are equal; also in the 2nd-order solution they are equal for the two methods. (iii) It is observed that the Reynolds number $R$ is proportional directly to inertia force and inversely to viscosity force.

Therefore, we have concluded that the considered methods can be used simply in various fields of science and engineering.

\section{Conflicts of Interest}

The authors declare that they have no conflicts of interest.

\section{References}

[1] W. Tan and T. Masuoka, "Stokes first problem for a second grade fluid in a porous half-space with heated boundary," International Journal of Non-Linear Mechanics, vol. 40, no. 4, pp. 515-522, 2005.

[2] M. H. Hamdan, "An alternative approach to exact solutions of a special class of navier-stokes flows," Applied Mathematics and Computation, vol. 93, no. 1, pp. 83-90, 1998.

[3] M. H. Hamdan and F. M. Allan, "A note on the generalized beltrami flow through porous media," International Journal of Pure and Applied Mathematics, vol. 27, no. 4, pp. 491-500, 2006.

[4] K. Vafai and C. L. Tien, "Boundary and inertia effects on flow and heat transfer in porous media," International Journal of Heat and Mass Transfer, vol. 24, no. 2, pp. 195-203, 1981. 
[5] S. Islam, M. R. Mohyuddin, and C. Y. Zhou, "Few exact solutions of non-newtonian fluid in porous medium with hall effect," Journal of Porous Media, vol. 11, no. 7, pp. 669-680, 2008.

[6] S. Islam and C. Y. Zhou, "Certain inverse solutions of a secondgrade magnetohydrodynamic aligned fluid flow in a porous medium," Journal of Porous Media, vol. 10, no. 4, pp. 401-408, 2007.

[7] K. Vafai, Hand Book of Porous Media, CRC Press, Taylor and Francis Group, 2005.

[8] M. J. Stefan, "Versuch uber die scheinbare adhasiom," Sitzungsberichte/Osterreichische Akademie der Wissenschaften in Wien. Mathematisch-Naturwissenschaftliche Klasse, vol. 69, pp. 713$721,1874$.

[9] J. F. Thorpe, Developments in Theoretical and Applied Mechanics, Pergamon Press, Oxford, UK, 1976.

[10] P. S. Gupta and A. S. Gupta, "Squeezing flow between parallel plates," Wear, vol. 45, no. 2, pp. 177-185, 1977.

[11] R. L. Verma, "A numerical solution for squeezing flow between parallel channels," Wear, vol. 72, no. 1, pp. 89-95, 1981.

[12] P. Singh, V. Radhakrishnan, and K. A. Narayan, "Squeezing flow between parallel plates," Ingenieur-Archiv, vol. 60, no. 4, pp. 274281, 1990.

[13] P. J. Leider and R. B. Byron, "Squeezing flow between parallel disks. I. theoretical analysis," Industrial and Engineering Chemistry Fundamentals, vol. 13, no. 4, pp. 336-341, 1974.

[14] M. Qayyum, H. Khan, M. T. Rahim, and I. Ullah, "Analysis of unsteady axisymmetric squeezing fluid flow with slip and no-slip boundaries using OHAM," Mathematical Problems in Engineering, Article ID 860857, 11 pages, 2015.

[15] M. Qayyum, H. Khan, M. T. Rahim, and I. Ullah, "Modeling and analysis of unsteady axisymmetric squeezing fluid flow through porous medium channel with slip boundary," PLoS ONE, vol. 10, no. 3, Article ID e0117368, 2015.

[16] A. A. Hemeda and E. E. Eladdad, "Iterative methods for solving the fractional form of unsteady axisymmetric squeezing fluid flow with slip and no-slip boundaries," Advances in Mathematical Physics, Article ID 6021462, 2016.

[17] M. Qayyum, H. Khan, and O. Khan, "Slip analysis at fluidsolid interface in MHD squeezing flow of casson fluid through porous medium," Results in Physics, vol. 7, pp. 732-750, 2017.

[18] I. Ullah, M. T. Rahim, H. Khan, and M. Qayyum, "Analytical analysis of squeezing flow in porous medium with MHD effect," University of Bucharest Scientific Bulletin Series A. Applied Mathematics and Physics, vol. 78, no. 2, pp. 1223-7027, 2016.

[19] I. Ullah, M. T. Rahim, H. Khan, and M. Qayyum, "Analytic comparison of MHD squeezing flow in porous medium with slip condition," Physics Research International, vol. 2016, Article ID 5407916, 10 pages, 2016.

[20] O. Khan, M. Qayyum, H. Khan, and M. Ali, "Improved analysis for squeezing of newtonian material between two circular plates," Advances in Materials Science and Engineering, vol. 2017, Article ID 5703291, 9 pages, 2017.

[21] R. Kandasamy, N. A. bt Adnan, and R. Mohammad, "Nanoparticle shape effects on squeezed MHD flow of water based $\mathrm{Cu}$, $\mathrm{Al}_{2} \mathrm{O}_{3}$ and SWCNTs over a porous sensor surface," Alexandria Engineering Journal.

[22] A. Madaki, R. Roslan, M. Rusiman, and C. Raju, "Analytical and numerical solutions of squeezing unsteady $\mathrm{Cu}$ and $\mathrm{TiO}_{2}$ nanofluid flow in the presence of thermal radiation and heat generation/absorption," Alexandria Engineering Journal.
[23] T. Hayat, T. Muhammad, A. Qayyum, A. Alsaedi, and M. Mustafa, "On squeezing flow of nanofluid in the presence of magnetic field effects," Journal of Molecular Liquids, vol. 213, pp. 179-185, 2016.

[24] S. Tauseef Mohyud-Din, U. Khan, and M. H. Saleh, "Numerical investigation of MHD flow and heat transfer of copper- water nanofluid in a channel with non- parallel walls considering different shapes of nanoparticles," Advances in Mechanical Engineering, vol. 8, no. 3, pp. 1-9, 2016.

[25] U. Khan, N. Ahmed, and S. T. Mohyud-Din, "Heat transfer effects on carbon nanotubes suspended nanofluid flow in a channel with non-parallel walls under the effect of velocity slip boundary condition: a numerical study," Neural Computing and Applications, pp. 1-10, 2015.

[26] I. K. Youssef and H. A. El-Arabawy, "Picard iteration algorithm combined with gauss-seidel technique for initial value problems," Applied Mathematics and Computation, vol. 190, no. 1, pp. 345-355, 2007.

[27] E. A. Ibijola and B. J. Adegboyegun, "A comparison of Adomians decomposition method and Picard iteration method in solving nonlinear differential equations," Global Journal of science Frontier Research F: Mathematices and Decision Sciences, vol. 12, no. 7, 2012.

[28] A. A. Hemeda, "Iterative methods for solving fractional gas dynamics and coupled burgers' equations," International Journal of Mathematical Modelling and Numerical Optimisation, vol. 6, no. 4, pp. 282-298, 2015.

[29] A. A. Nahla and A. A. Hemeda, "Picard iteration and padé approximations for stiff fractional point kinetics equations," Applied Mathematics and Computation, vol. 293, pp. 72-80, 2017.

[30] Z. Odibat and S. Momani, "Numerical methods for nonlinear partial differential equations of fractional order," Applied Mathematical Modelling, vol. 32, no. 1, pp. 28-39, 2008.

[31] T. Özis and A. Yildirim, "Comparison between adomain method and hes homotopy perturbation method," Applied Mathematics and Computation, vol. 56, no. 5, pp. 1216-1224, 2008.

[32] A.-M. Wazwaz, "A new algorithm for calculating adomian polynomials for nonlinear operators," Applied Mathematics and Computation, vol. 111, no. 1, pp. 53-69, 2000.

[33] S.-P. Yan, H. Jafari, and H. K. Jassim, "Local fractional adomian decomposition and function decomposition methods for laplace equation within local fractional operators," Advances in Mathematical Physics, vol. 2014, Article ID 161580, 7 pages, 2014.

[34] D. Baleanu, J. A. Tenreiro Machado, C. Cattani, M. C. Baleanu, and X.-J. Yang, "Local fractional variational iteration and decomposition methods for wave equation on cantor sets within local fractional operators," Abstract and Applied Analysis, vol. 2014, Article ID 535048, 6 pages, 2014.

[35] I. Podlubny, Fractional Differential Equations, vol. 198 of Mathematics in Science and Engineering, Academic Press, San Diego, Calif, USA, 1999.

[36] M. Caputo, Elasticita E Dissipazione, Zani-Chelli, Bologna, Italy, 1969. 


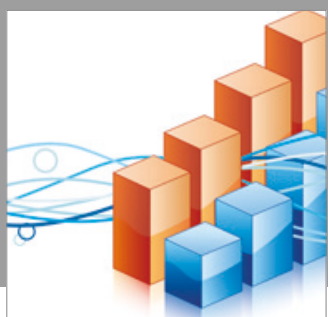

Advances in

Operations Research

vatersals

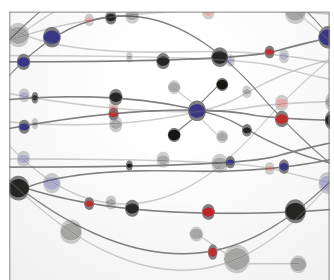

\section{The Scientific} World Journal
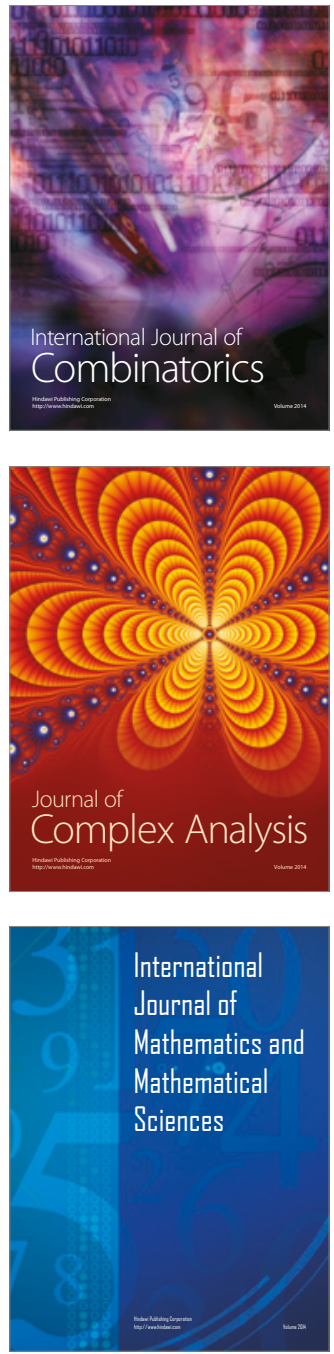
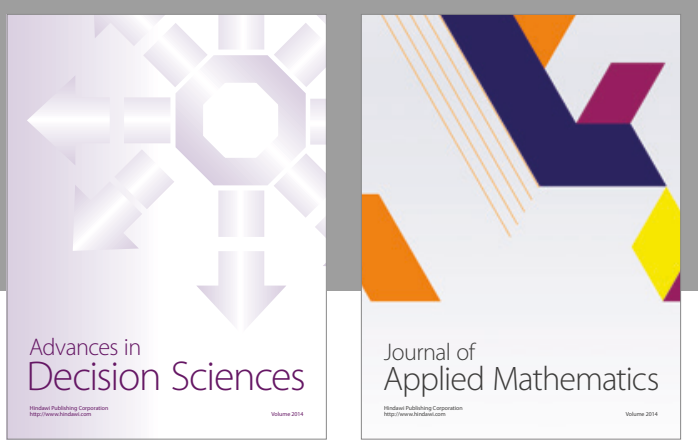

Algebra

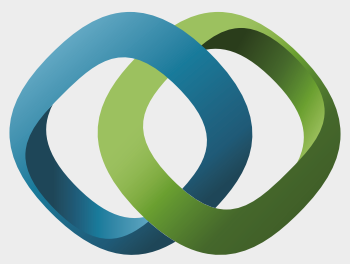

\section{Hindawi}

Submit your manuscripts at

https://www.hindawi.com
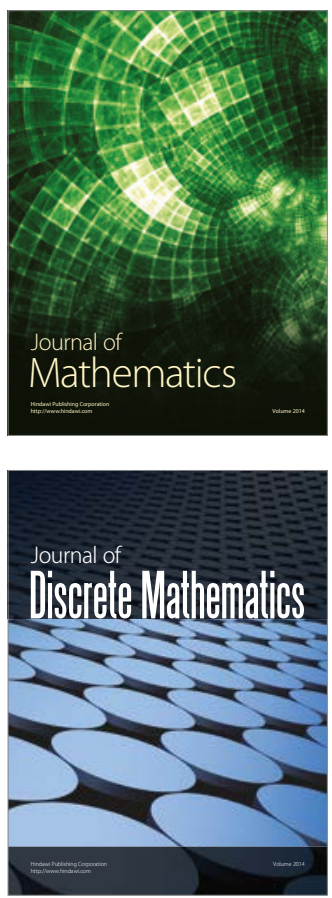

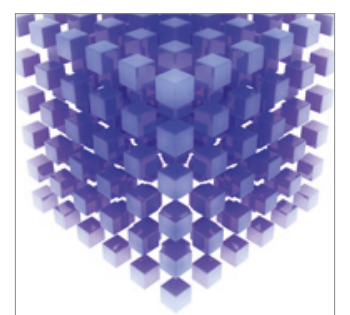

Mathematical Problems in Engineering
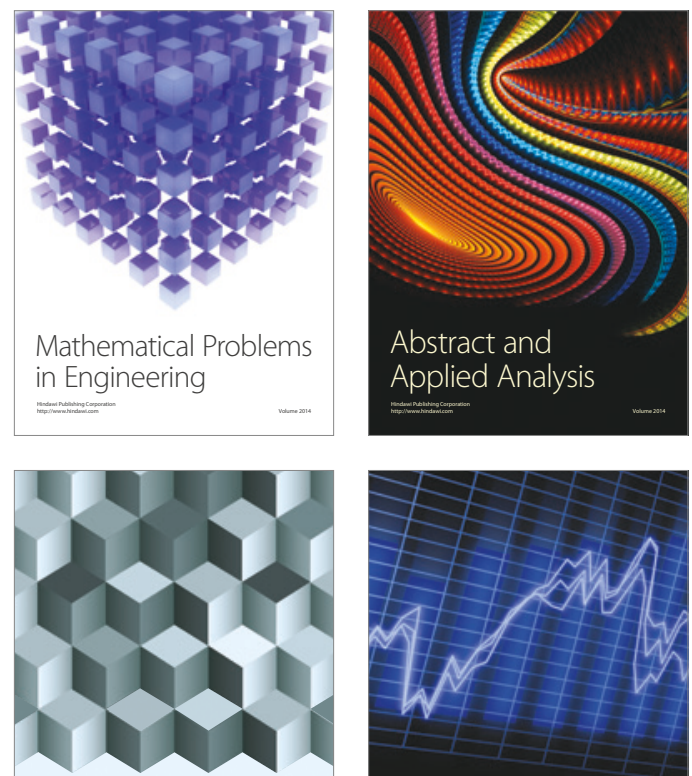

Journal of

Function Spaces

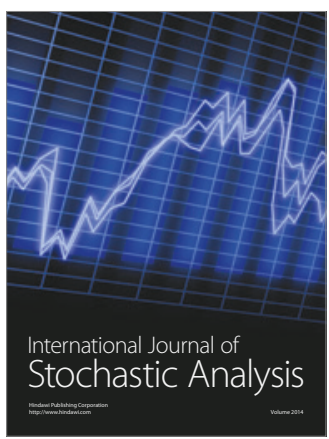

Probability and Statistics
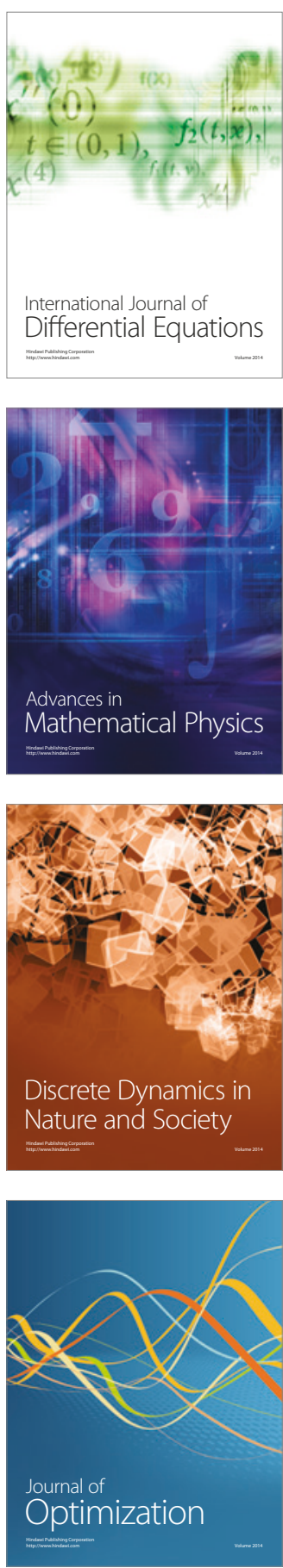